\title{
Hierarchical pseudo-ductile hybrid composites combining continuous and highly aligned discontinuous fibres
}

$\mathrm{HaNa} \mathrm{Yu}^{* 12}$, Marco L. Longana ${ }^{2}$, Meisam Jalalvand ${ }^{23}$, Michael R. Wisnom², Kevin D. Potter ${ }^{2}$

${ }^{1}$ Department of Mechanical Engineering, University of Bath, Bath, BA2 7AY, UK

2 Bristol Composites Institute, Advanced Composites Collaboration for Innovation and Science, University of Bristol, Queen's Building, University Walk, Bristol, BS8 1TR, UK

3 Department of Mechanical and Aerospace Engineering, University of Strathclyde, 75 Montrose Street, Glasgow, G1 1XJ, UK

*Corresponding author,E-mail: H.Yu@bath.ac.uk,Hana.Yu@bristol.ac.uk

Keywords: A. Discontinuous reinforcement; A. Hybrid; C. Analytical modelling; D. Failure

\section{Abstract}

Hybrid composites allow avoiding catastrophic failure, a key limitation of composite materials, and can provide a balanced suite of modulus, strength and ductility. The aim of this research is to manufacture hierarchical hybrid composites using a combination of continuous high elongation fibres and intermingled hybrids made out of highly aligned discontinuous fibres with lower elongation to achieve pseudo-ductility through control of failure development. The HiPerDiF (High Performance Discontinuous Fibres) method that allows a high level of fibre alignment, leading to excellent mechanical properties close to continuous fibre composites, was used to produce the intermingled hybrid discontinuous fibre preforms. The hierarchical hybrid composite configuration is composed of an intermingled hybrid discontinuous fibre layer sandwiched between continuous S-glass layers. The overall stress-strain response of the intermingled hybrid composites and the hierarchical hybrid composites was investigated for different fibre types and ratios. The analytical modelling approach previously developed by the authors for interlaminated hybrid composites was 
modified for this new type of hierarchical composite. The experimental results were analysed and the analytical model was used to evaluate the optimised balance of constituents to maximise pseudo-ductile strain in tension.

\section{Introduction}

High performance composites are typically made of continuous fibres embedded in a polymer matrix but, unlike metals, they tend to fail in a brittle manner leading to difficulties in designing structural components $[1,2]$. Hybridising two or more types of fibres in composites is one of the main approaches to overcome catastrophic failure.

Several researchers investigated the mechanical properties and damage modes of hybrid composites with continuous and discontinuous fibres as a function of the ratio of constituents and dispersion state. Based on the distribution of each constituent, hybrid composites are categorised into three major types, interlaminated, intraply, and intermingled hybrids, as reported and summarised in $[3,4]$. However, as the interlaminated hybrid configuration is easily achievable with the material forms available on the market, most of the available literature is mainly focused on this type of hybrid, where the hybridisation is achieved at lamina level by stacking plies of different constituents [3, 5-7]. Yarn-by-yarn hybrid fabric prepregs are also commercially available. These give specific characteristics to composite materials, e.g. carbon/aramid hybrid fabric for protection against bullets [8] and carbon/metal hybrid fabric for electric shielding, with mechanical properties reported in [9].

Highly aligned discontinuous fibre composites allow high design freedom in the hybridisation level while achieving structural performance comparable with continuous fibre reinforced composites when the fibre aspect ratio is sufficiently high to achieve full load transfer [10]. Several flow-induced alignment techniques have achieved some success with a high fibre alignment level and a uniform fibre dispersion state in the intermingled hybrids, e.g. the MBB-VTF (Vacuum-drum-filter) alignment process, which relies on the high shear stress 
of a viscous liquid medium, such as glycerine, to align fibres [10-13]. The authors concluded that interlaminated and intermingled hybrid composites with the same carbon/glass ratio show approximately the same level of elastic modulus and impact strength but the intermingled hybrid composite was largely superior in flexural and tensile strength. More recently, $\mathrm{Yu}$ and Longana et al. $[14,15]$ investigated the effects of the fibre mixing ratio on the overall stress-strain responses of intermingled discontinuous glass/carbon and recycled/virgin carbon fibre hybrid composites manufactured with the HiPerDiF (High Performance Discontinuous Fibre) method. The HiPerDiF method, invented at the University of Bristol, relies on a unique fibre orientation mechanism leading to high mechanical performance, as outlined in [16]. Thanks to the fact that a low viscosity medium, i.e. water, is used instead of the high viscosity media used in conventional fibre alignment methods, the HiPerDiF technology has the potential to be a fast and continuous process to produce highly aligned tape type preforms. The prototype machine successfully produced intermingled hybrid composites with highly aligned discontinuous fibres that showed a high level of pseudo-ductility [14].

Although hybrid composites are one of the portfolios of next generation composites development, there are few papers about the possibility to hierarchically organise the material constituents $[17,18]$. The hierarchical organisation of hybrid composites constituents allows a flexible tailoring of the stress-strain curves by controlling the failure mechanism of each constituent. This paper proposes an example of a new type of hierarchically organised hybrid composite which is interlaminated and where some of the layers are also hybrids with aligned discontinuous fibre intermingled preforms sandwiched between continuous S-glass layers. As previously observed in [14], intermingled hybrid composites showed a brittle linear or pseudo-ductile nonlinear tensile response depending on the type of fibres and ratio. This paper demonstrates how the behaviour of the core material affects the tensile response of the 
interlaminated hybrid composite and the benefits of the hierarchical organisation on pseudoductility. The modelling approach developed by Jalalvand et al. [19] is further developed, applied to hierarchical hybrid composites and then validated with the experimental results.

\section{Analytical model}

Jalalvand's analytical model developed in [19] predicts the stress-strain curve of layer-bylayer hybrid composites with a low elongation material in the middle layer sandwiched between high elongation materials. The model offers three damage mode criteria: (i) fragmentation in the low elongation material; (ii) delamination between the low elongation material and high elongation material; (iii) failure in the high elongation material. The model also offers analytical predictions for the corresponding stress levels at which these damage modes are expected to occur.

In this paper, the hierarchical hybrid composites consist of three types of materials, i.e. the mixture of low and intermediate elongation materials (LE, IE) in the middle and the high elongation material (HE) in the outer layers as shown in Figure 1. The Jalalvand model was further developed to predict the stress-strain curve for hierarchical hybrid composites. The procedure for finding the damage process is shown in Figures 2 and 3 as the stress-strain curves of the intermingled hybrid composites in tension are categorised into two cases based on the mechanical properties of the low and intermediate elongation materials and their ratio in the hybrid; linear (elastic) and nonlinear (pseudo-ductile) behaviours.

\section{a. Linear-elastic behaviour of intermingled hybrids}

When the intermingled layer shows a linear stress-strain curve, the intermingled layer can be considered as a homogenised unit (Figure 2(a)). When the low elongation material fails in the intermingled layer, the intermediate elongation material cannot withstand the load therefore these instantly cause cracking of the intermingled layer and the appearance of a sequence of fragmentations over the specimen length. Its failure process therefore follows that of the 
interlaminated hybrid composites with 2 types of material as investigated in [19]. The tensile response of the hierarchically organised interlaminated hybrid composite can be predicted by comparing the fragmentation stress in the intermingled layer $(\sigma @ i t m F)$, the delamination stress between the intermingled layer and the high elongation material $(\sigma @ d e l)$, and the final failure stress of the high elongation material $\left(\sigma_{@ H F}\right)$. These stress levels denote the average stress in the laminate. As shown in Figure 2(b), the stress-strain responses can be drawn using the characteristic points given in Table 1 , connected by straight lines from the origin $(0,0)$ up to high elongation material failure [19]. In this paper, the stress at which the first crack occurs, $\sigma_{@ i t m F}$, in the intermingled layer was assumed to be the same as the stress at which fragmentation progresses, $\sigma @ f r a g$,

$$
\sigma_{@ i t m F}=\sigma_{@ f r a g}=S_{L} \frac{\alpha \beta+1}{\alpha(\beta+1)}
$$

where $\alpha$ and $\beta$ are the modulus and thickness ratios of the intermingled layer to the high elongation composites respectively $\left(\alpha=\frac{E_{L+I}}{E_{H}}, \beta=\frac{t_{i t m}}{t_{H}}\right) ; E_{L+I}$ is the elastic modulus of intermingled layer made of low and intermediate elongation materials and $E_{H}$ is the elastic modulus of high elongation material, $t_{i t m}$ and $t_{H}$ denote the half thickness of the intermingled layer and high elongation materials. In order to calculate the strain of the saturated fragmentation knee point (P3 in the case of L2) and L3) in Table 1 and Figure 2(b)), the modulus of the laminate with randomly saturated fragmentation in the intermingled layer, $E_{\text {sat }}$, [19] is derived as:

$$
E_{\text {sat }}=E_{H} \frac{1+\alpha \beta}{(1+\beta)\left(1+\frac{11}{18} \alpha \beta\right)}
$$

$\sigma @ d e l$ is calculated using Equation (3) in an aspect of fracture mechanics and $\sigma_{@ H F}$ using Equation (4) respectively [19],

$$
\sigma_{@ d e l}=\frac{1}{1+\beta} \sqrt{\frac{1+\alpha \beta}{\alpha \beta} \frac{2 G_{I I C} E_{H}}{t_{H}}}
$$




$$
\sigma_{@ H F}=\frac{S_{H}}{K_{t}(1+\beta)}
$$

where $G_{I I c}$ is the mode II fracture toughness and $S_{H}$ is the high elongation material failure strength. The stress concentration factor, $K_{t}$, is assumed around the interlaminar crack tip. In addition to the catastrophic failure process case that occurs when the high elongation material failure stress $(\sigma @ H F)$ is lower than the intermingled layer failure or fragmentation stress $(\sigma @ i t m F)$, three different types of the failure development can be defined as shown in Figure 2(b). The analytical solutions for $\sigma_{@ i t m F}, \sigma_{@ d e l}$, and $\sigma_{@ H F}$ are fully derived in [19].

\section{b. Nonlinear behaviour of intermingled layer}

The fragmentation of the low elongation fibres does not lead to the rupture of the whole intermingled layer when the intermingled hybrid composites show nonlinear behaviour in their stress-strain curves. The model was therefore modified to have a 2-step fragmentation development, predicting the first knee from the low elongation material fragmentation and the second knee from the intermingled layer fragmentation and determining whether delamination occurs or not at the second knee point. Each characteristic point was calculated using the equations summarised in Table 2, however, catastrophic failure or unstable delamination failure processes were not included.

In the first step, the intermediate and high elongation materials are considered as a homogenised unit except the low elongation material. The stress in the laminate at which the first crack in the low elongation material occurs, $\sigma @ L F$, was calculated using Equation (5) in [19]:

$$
\sigma_{@ L F}=\sigma_{@ f r a g 1}=S_{L} \frac{\alpha_{1} \beta_{1}+1}{\alpha_{1}\left(\beta_{1}+1\right)}
$$

where $S_{L}$ is the low elongation material strength $\left(S_{L}=\varepsilon_{L F} E_{L}\right), \alpha_{I}$ is the modulus ratio of the low elongation material $\left(E_{L}\right)$ to the intermediate and high elongation materials hybrid composites $\left(E_{I+H}\right)$. Since the dimension of the individual fibres (7-12 $\mu \mathrm{m}$ diameter usually) in the intermingled layer is small, the energy release rate is low, so no delamination is expected 
to follow the first knee after the low elongation material fragmentation, as also described in Figure 3 (a). $\beta_{1}$ is the ratio of the volume of low elongation material $\left(V_{L}\right)$ to the volume of intermediate and high elongation materials hybrid composites $\left(V_{I}+V_{H}\right)[14] . E_{\text {sat } 1}$ denotes the modulus of the laminate with randomly saturated fragmentation in the low elongation material:

$$
E_{\text {sat } 1}=E_{I+H} \frac{1+\alpha_{1} \beta_{1}}{\left(1+\beta_{1}\right)\left(1+\frac{11}{18} \alpha_{1} \beta_{1}\right)}
$$

The strain at which the intermediate elongation material fails after fragmentation saturation of the low elongation material (P4 in the case of N1) and N2) in Figure 3(b) and Table 2) is

$$
\varepsilon_{@ I F-P S}=\varepsilon_{I F}-\frac{7}{18} \frac{S_{L} \beta_{1}}{E_{I+H}}
$$

The first term in this equation is the intermediate elongation material failure strain, $\varepsilon_{I F}$, and the second term shows the amount of strain reduction due to the load carried by the low elongation material, as explained in [19]. $\varepsilon @ I F-P S$ also represents the strain at which the intermingled layer fracture or fragmentation starts in the second step. Consequently, $\sigma_{@ i t m F}$, the stress at $\varepsilon @ I F-P S$, is calculated to be

$$
\sigma_{@ i t m F}=\varepsilon_{@ I F-P S} E_{I+H}\left(V_{I}+V_{H}\right)
$$

This is the same as the stress at which intermingled layer fragmentation progresses, $\sigma @ f r a g 2$. The strain after the intermingled layer fragmentation at the second knee, $\frac{\sigma_{@ \text { frag } 2}}{E_{\text {sat } 2}}, \mathrm{P} 5$ in Figure 3(b) and Table 2, is also calculated defining the modulus of laminate with randomly saturated fragmentation in the intermingled layer, $E_{\text {sat2 }}$, as:

$$
E_{\text {sat } 2}=E_{H} \frac{1+\alpha_{2}+\alpha_{2} \beta_{2}}{\left(1+\beta_{2}\right)\left(1+\frac{11}{18} \alpha_{2} \beta_{2}\right)}
$$

where $\alpha_{2}$ is the modulus ratio of the intermingled layer $\left(E_{I} V_{I}\right)$ to the high elongation material $\left(E_{H}\right)$ and $\beta_{2}$ is the thickness ratio of the intermingled layer to the high elongation material, $\frac{t_{i t m}}{t_{H}}$. The modulus of the intermingled layer is reduced after the low elongation material fragments, while its thickness does not change. The whole intermingled layer can be assumed 
to be delaminated after the intermediate fibres initiated intermingled layer fragmentation at the second knee point when the delamination stress, $\sigma_{@ d e l}$, is less than the strength of the high elongation material, $\sigma @ H F$, as illustrated in Figure 3(b), N2) case. $\sigma_{@ d e l}$ is also calculated using Equation (10) [19].

$$
\sigma_{@ d e l}=\frac{1}{1+\beta_{2}} \sqrt{\frac{1+\alpha_{2} \beta_{2}}{\alpha_{2} \beta_{2}} \frac{2 G_{I I C} E_{H}}{t_{H}}}
$$

\section{Experimental}

To manufacture intermingled hybrid preforms, the HiPerDiF (High Performance Discontinuous Fibre) method was used. The fibre orientation mechanism of the HiPerDiF method [16], in Figure 4, is simply summarised as follows. A suspension of fibre dispersed in water is supplied, through a peristaltic pump, to the fibre orientation unit, i.e. two parallel plates at a controlled distance, $d$. Provided that the fibre length is less than $d$, when the suspension jet hits the furthermost plate at an angle, the fibres are aligned by a sudden momentum change. The fibres then fall onto a conveyor mesh belt where the alignment is finalised, a vacuum suction line underneath the mesh belt removes the water, creating a dry fibre preform. A lab-scale machine (Figure 5), capable of aligning discontinuous fibres, drying the remained water with an infrared lamp and delivering tape type preforms with up to $5 \mathrm{~mm}$ in width to a resin film impregnation stage with an in-line process, has been designed and built for high productivity [20].

\subsection{Materials and manufacturing condition}

\section{a. Intermingled hybrid composites}

The scaled-up HiPerDiF machine allows manufacturing of a range of intermingled hybrid composites. In this paper, intermingled hybrid composites were manufactured with different fibre mixing ratios in order to study the effect of hybridisation on the tensile modulus and strength, and shape of the stress-strain curve under the same manufacturing condition (Benchmark machine parameters are listed in Table 3). The properties of the used fibres, i.e. 
high tensile strength carbon fibre (HSC, C124, TohoTENAX), high tensile modulus carbon fibres (HMC, Granoc XN-90, NGF) and E-glass fibres (EG, C100, Vetrotex), are listed in Table 4. Three sets of intermingled hybrid specimens were manufactured: HMC/EG and HMC/HSC with $0 \%, 20 \%, 40 \%, 50 \%, 60 \%$ and $80 \%$ HMC fibre content, and HSC/EG with $0 \%, 20 \% 40 \%, 60 \%, 80 \%$ and $100 \%$ HSC fibre content. It has to be remarked that, as found in [14], it is not possible to manufacture $100 \%$ HMC specimens. The HMC fibres are particularly difficult to handle because of their non-sized surface, which causes the fibres to be statically charged, compromising the alignment level during the water drying process with the infrared lamp.

The Aligned Discontinuous fibre preforms (AD) partially impregnated with an epoxy resin film (MTM49-3, Cytec) were laid-up in a semi-closed mould with the stacking sequence of $\left[\mathrm{AD}_{4}\right]$, placed in a vacuum bag $(1 \mathrm{bar})$ and then cured in an autoclave at $135^{\circ} \mathrm{C}$ for 135 minutes at 6 bar. The autoclave pressure was applied at the beginning of the curing cycle. The nominal thickness of the intermingled hybrid composites is $0.22 \mathrm{~mm}$. A fibre volume fraction of approximately $35-40 \%$ was estimated by taking into account the aligned fibre preform and resin film areal weight ratio and the thickness of the cured composite specimens measured from microscopy images. The areal weight of the resin film, $80 \mathrm{~g} / \mathrm{m}^{2}$, is significantly higher than the one of the aligned fibre preform, $50-70 \mathrm{~g} / \mathrm{m}^{2}$ : it is foreseen that using lower areal weight resin films will significantly increase the fibre volume fraction, as shown in [14].

\section{b. Hierarchical hybrid composites}

Interlaminated hybrid composite specimens were laid up by sandwiching intermingled hybrid discontinuous fibre preforms, manufactured with the HiPerDiF method as described in Section 3.1a, between continuous unidirectional S-glass prepreg plies as shown in Figure 1. The stacking sequence of the laminates was $\left[\mathrm{cSG}_{2} / \mathrm{AD}_{2} / \mathrm{cSG}_{2}\right]$ where $\mathrm{cSG}$ denotes a continuous S-glass layer. For the AD layers, dry intermingled hybrid fibre preforms with the 
same fibre mixing ratios described above were used. The laminates were placed in a mould and cured by vacuum bag moulding in an autoclave under the same conditions described above, the resin excess from the cSG was used to impregnate the AD. Fibre volume fractions for the $\mathrm{AD}$ and cSG layers were approximately $35-40 \%$ and $65 \%$ respectively. The nominal thickness of the interlaminated hybrid composites is $0.56 \mathrm{~mm}$ with the thickness ratio of ADcSG being 1:4.

\subsection{Specimen preparation and tensile test method}

Glass fibre reinforced plastic end-tabs were bonded with Huntsmann Araldite 2014-1. A schematic of the specimen with dimensions is shown in Figure 6. Tensile tests were carried out using a servo electric testing machine (Shimadzu, $10 \mathrm{kN}$ ) with a cross-head displacement speed of $1 \mathrm{~mm} / \mathrm{min}$. A white speckle pattern over a black background was painted on the specimens to allow the strain measurement with a video extensometer (IMETRUM). The gauge length for the strain measurement was approximately $40 \mathrm{~mm} .3$ specimens were used per each test set for all the cases.

\section{Tensile test results}

\subsection{Intermingled hybrid composites}

Representative stress-strain curves for the three sets of specimens are shown in Figure 7(a)(c).

When the HSC fibres are intermingled with the EG fibres, Figure 7(a), no plateau is generated as the low elongation fibre (HSC fibre) failure is immediately followed by the whole composite specimen rupture.

The intermingled composites where the low elongation fibres are the HMC fibres, Figures 7(b) and (c), showed a clear pseudo-ductile behaviour: for HMC fibre contents between 20\% and $60 \%$ a plateau is observable, as will be discussed in Section 5.1. 
The relevant information obtained from the tests are summarised in Tables from 5 to 7 . The pseudo-ductile properties of each case were measured based on the definition suggested by Wisnom et al. [21]. The yield stress, Yield $\sigma_{11}$, is defined as the intersection of the stressstrain curve and a straight line with gradient $E_{11}$ and $0.1 \%$ offset from the origin, this is equivalent to the definition of proof stress in metals. Where applicable, the Knee $\varepsilon_{11}$ is determined from the intersection of the two linear regions, i.e. the linear-elastic region and the fragmentation plateau, which is equivalent to the definition of transition strain described in ASTM standard D3039 [22]. Failure $\sigma_{11}$ and $\varepsilon_{11}$ are the values of stress and strain at which the specimens lose their integrity. The pseudo-ductile strain, $\varepsilon_{p s d}$, is the difference between the Failure $\varepsilon_{11}$ and the elastic strain at the same stress level based on the initial modulus, $E_{11}$.

\subsection{Hierarchical hybrid composites}

Figure 8(a)-(c) shows representative stress-strain curves for the three sets of specimens.

A pseudo-ductile behaviour appeared in the intermingled HSC/EG with continuous S-glass composites, Figure 8(a), caused by the intermingled layer fragmentation and delamination, as will be discussed in Section 5.2.

The intermingled HMC/EG with continuous S-glass composites, Figure 8(b), also show a nonlinearity due to the HMC fibre fragmentation. Since the HMC fibre failure did not result in the fragmentation of the intermingled layer and the discontinuous E-glass fibres in the intermingled layer were separated by the continuous S-glass from the end-tabs, avoiding stress concentrations or premature failure, the E-glass fibres did not fail around $2 \%$ as they did in the all discontinuous E-glass composite case, Figures 7(a) and (b). The contemporaneous E-glass fibre fracture and the S-glass fibre splitting are identified as the cause of the nonlinear behaviour after $3.3 \%$ of strain. 
The hierarchical hybrid composites with the HMC/HSC intermingled layer, Figure 8(c), show two distinct knee points, which, to the best knowledge of the authors, was not observed in other types of hybrids before.

The relevant information obtained from the tests is summarised in Tables from 8 to 10. For most cases, the tests were interrupted at 3.3\% strain in order to preserve the fragmentation and delamination status in the specimen. The Failure $\sigma_{11}$ is therefore defined as the stress level at $3.3 \%$ strain and the pseudo-ductile strain, $\varepsilon_{p s d}$, is the difference between $3.3 \%$ and the elastic strain at the same stress level based on the initial modulus, $E_{11}$.

\section{Discussion}

\subsection{Intermingled hybrid composites}

In the case of the HSC/EG, straight stress-strain curves can be observed as shown in Figure 7(a), this is caused by the fact that no substantial HSC fibre fragmentation happens before the failure of the whole intermingled hybrid specimen. The failure is catastrophic mainly because the failure strain of the all HSC composite, $1.47 \%$, is relatively close to the failure strain of the all EG composite, $1.94 \%$, therefore, when the two fibres are mixed, the obtained intermingled hybrid composites do not show a plateau region. When the difference of the failure strain values between the two fibres type is substantial, i.e. $\mathrm{HMC} / \mathrm{EG}$, Figure 7(b) and HMC/HSC, Figure 7(c), a clear pseudo-ductile behaviour can be observed. However, it must be underlined that for $\mathrm{HMC}$ fibre contents higher than $60 \%$, the failure is brittle, this is caused by the fact that the stress released when the HMC fibres fail cannot be borne by the higher elongation fibres (HSC or EG). Therefore, the amount of low elongation fibres (HMC) dictates whether there is any nonlinear behaviour. In this paper, the low elongation material is stiffer than the higher elongation material and bears the higher amount of stress: its content not only affects the initial modulus but also the shape of the transition between the elastic deformation and the fragmentation plateau. In the specimens with low content of the HMC 
fibres, most of the fibres are surrounded by high elongation fibres (HSC or EG fibres) and therefore fragment individually; this results in a very smooth transition between the linear elastic part of the curve and the fragmentation plateau. When the low elongation fibre ratio is increased, the transition between the elastic region and the fragmentation plateau becomes sharper. It is relevant to note that, for both $\mathrm{HMC} / \mathrm{EG}$ and $\mathrm{HMC} / \mathrm{HSC}$ intermingled hybrids with a HMC fibre content of $60 \%$, the fragmentation plateaus are not followed by a second linear phase with increasing stress but are interrupted by the specimen failure. As mentioned above, it is possible, for the $\mathrm{HMC} / \mathrm{EG}$ and $\mathrm{HMC} / \mathrm{HSC}$ intermingled specimens, to evaluate the pseudo-ductile strain. By observing Figure 9, it can be concluded that the pseudo-ductile strain presents a maximum value for the HMC fibre content of $40 \%$ in the $\mathrm{HMC} / \mathrm{EG}$ composite with $270 \mathrm{MPa}$ Yield $\sigma_{11}$. Maximum pseudo-ductility is at 50\% $\mathrm{HMC}$ in the HMC/HSC composite with a yield stress of $400 \mathrm{MPa}$. Figure 10 shows the failure stress of the three sets of specimens as a function of the low elongation fibres content with the linear Rule of Mixtures (RoM), bilinear RoM and its construction lines. Interestingly, the ultimate failure stresses of the composites with pseudo-ductile behaviour (HMC/EG and HMC/HSC) decrease with the increase of the HMC fibre amount. On the other hand, the HSC/EG hybrid composites that fail in a brittle manner show a trend that follows the bilinear rule of mixtures based on a constant strain assumption, where all of the low elongation fibres in the hybrid composite break at their failure strain, followed by failure of the high elongation fibres [4]. This difference is a direct consequence of the completely different behaviour of the two sets of specimens: for the specimens that present a pseudo-ductile behaviour the damage begins with the fragmentation of the HMC fibres while for the HSC/EG intermingled hybrids the failure is brittle and both the fibre types fail at the same time.

The increase of failure strain of the low elongation fibres in hybrid composites compared to all-low elongation fibre composites is called the 'hybrid effect' [23, 24]. A recent review 
paper [4] reported the failure development process to be the main hypothesis for the cause of the hybrid effect. Combining two different types of fibre can significantly alter the development of the critical cluster of fibre breaks and thereby increase the failure strain of the low elongation fibres in the hybrid composite. There are many parameters that may influence the hybrid effect: the fibre dispersion, the low elongation fibre strength scatter, the failure strain ratio, the relative volume fraction, and the fibre modulus ratio. Comparing the intermingled $\mathrm{HMC} / \mathrm{EG}$ and $\mathrm{HMC} / \mathrm{HSC}$ composites cases, the first two parameters can be assumed to be the same and, in both cases, the failure strain ratio is higher than 2 , which should have negligible influence on the hybrid effect [25]. In this study, therefore, the relative volume fraction and the modulus ratio of the fibres are the variables driving the hybrid effect. Figure 11(a) shows the measured failure strain of the low elongation material at the knee point, Knee $\varepsilon_{11}$, of $\mathrm{HMC} / \mathrm{EG}$ and $\mathrm{HMC} / \mathrm{HSC}$ composites and Figure 11(b) compares their stress-strain curves as a function of the HMC content ratio, respectively. It was not possible to manufacture all HMC fibre composites due to the difficulty in handling the HMC fibres as described in Section 3.1a. However, as the intermingled hybrid composites with $80 \%$ HMC fibre content failed in a brittle manner, the all HMC fibre composite failure strain can be expected to be $0.2 \%$. In these two hybrid composites, the EG and HSC fibres play the role of high elongation fibres with the HSC fibre modulus $(225 \mathrm{GPa})$ much higher than that of the EG fibre (73 GPa); the HMC/HSC composites showed a higher hybrid effect than the HMC/EG composites independently from the relative volume content of HMC fibres. The influence of the high elongation fibre modulus on the hybrid effect is shown more clearly at high relative volume contents of low elongation fibres (HMC): the apparent failure strain of the $\mathrm{HMC} / \mathrm{HSC}$ composite at $60 \%$ of $\mathrm{HMC}$ content shows an increase of $20 \%$ compared to the HMC/EG composite. This is attributed to the decrease of the stress concentrations on the intact low elongation fibres (HMC) during the failure development, as well recognised in [25]. 
As the high elongation fibre modulus decreases, the stress concentration factor on the intact high elongation fibres drastically increases [26, 27]. However, the high relative volume fraction of high elongation fibres delays the development of critical clusters of low elongation fibres which can propagate and lead to a visible slope change in the stress-strain curve. This causes a slight hybrid effect increase driven by the high elongation fibre modulus at low relative volume fraction of low elongation fibres when comparing the HMC/EG and HMC/HSC composites.

\subsection{Hierarchical hybrid composites}

The stress-strain curves of the intermingled hybrid composites in tension are categorised into two cases; linear (elastic) and nonlinear (pseudo-ductile) behaviours. The former includes all the $\mathrm{HSC} / \mathrm{EG}$ intermingled hybrid composites and the $\mathrm{HMC} / \mathrm{EG}$ and $\mathrm{HMC} / \mathrm{HSC}$ intermingled hybrid composites with $80 \%$ HMC fibre content. The rest of the cases, i.e. the HMC/EG and HMC/HSC intermingled hybrid composites with 20, 40 and 60\% HMC fibre content, correspond to the latter non-linear case with pseudo-ductile response. The moduli of the HSC and EG composite materials were experimentally measured as listed in Table 5. The modulus of the $\mathrm{HMC}$ composite material was estimated as $260 \mathrm{GPa}$, assuming that the fibre volume is $35 \%$ and the fibre length and alignment factor is 0.85 as previously in [14]. The fragmentation strain of the low elongation material, $\varepsilon_{L F}$, was assumed to be the same as each fibre failure strain, i.e. $\mathrm{HSC}=1.93 \%, \mathrm{HMC}=0.398 \%$. To determine the properties of the continuous S-glass composite material in Table 11, pure S-glass specimens were manufactured with the same procedure and curing conditions as the hybrid composites. In order to calculate the delamination stress, $\sigma_{@ d e l}$, between the intermingled layer and the Sglass layer, $G_{I I C}$ was assumed to be $1.00 \mathrm{~N} / \mathrm{mm}$. To keep the analysis is simple and consistent,

the stress concentration factor, $K_{t}$, was assumed to be 1.08 which is equal to the value used in the previous analytical study [19]. It has to be also remarked that the thermal residual stress 
due to the mismatch of thermal expansion of each material during the curing process was neglected in the analytical model. This is because the compressive residual strain in the carbon layer (HSC) was found to be $2.9 \times 10^{-4} \mathrm{~m} / \mathrm{m}$ which is less than $2 \%$ of the strain to failure of the carbon fibres [21] therefore it does not affect the model and the failure process of the hybrid laminates significantly.

\section{a. Linear-elastic behaviour of intermingled hybrids}

The predicted and experimental results are shown in Figure 12. The model predicts the HSC/EG-cSG hybrid composites with $20,40,60 \%$ of HSC content in the intermingled layer to show fragmentation of the intermingled layer first and dispersed delamination between the intermingled layer and the S-glass layers to follow. The experimental curves and the corresponding pictures of the specimen surfaces captured at 3.3\% strain in Figure 13 show evidence of the fragmentation and delamination. However, the onset point of the intermingled layer failure was delayed, in particular in the lower HSC content case. This is attributed to the E-glass material altering the failure development, avoiding the development of HSC fibre critical clusters, producing the hybrid effect as described in the previous section. Also the strength variation in the intermingled hybrid layer was not taken into account in the analytical model therefore the experimental curves showed a smooth knee point. For the HSC/EG-cSG hybrid composite with $80 \%$ HSC content in the intermingled layer, the delamination stress was calculated to be slightly lower than the fragmentation stress level; therefore the model predicted a small load drop and single delamination. The delamination and fragmentation stresses were predicted to be close, and experimentally the load drop was not observed. The material showed a longer plateau with a limited number of cracks and a large delamination area as shown in Figure 13.

\section{b. Nonlinear behaviour of intermingled layer}


Figures 14(a) and (b) show the obtained experimental stress-strain curves compared with the results from the analytical model. As predicted by the model, the HMC/EG-cSG composites with $20,40,60 \%$ of $\mathrm{HMC}$ content in the intermingled layer and the HMC/HSC-cSG composites with $60 \%$ of $\mathrm{HMC}$ content in the intermingled layer did not show delamination as the stress required to cause delamination between the intermingled layer and cSG layers is higher than the stress at which the high elongation material (cSG) fails. This is clearly visible in Figure 15(a) and (b). For the HMC/EG-cSG composites with 20, 40, $60 \%$ of HMC content no cracks or delaminated areas can be observed. For the HMC/HSC-cSG composites with $60 \%$ of HMC content in the intermingled layer it is possible to observe some cracks but not the bright yellow areas representative of delaminated areas between the intermingled and S-glass layers. The model predicted that the HMC/HSC-cSG hierarchical composites with $20 \%$ and $40 \%$ of HMC fibre content in the intermingled layer show a dispersed delamination between the intermingled layer and the S-glass layers. This is in agreement with the experimental results. Dispersed delamination was also observed on the surface of the specimens as shown in Figure 15(b).

However, the HMC/EG-cSG and HMC/HSC-cSG hybrid composites with 80\% HMC fibre content in the intermingled layer showed an interesting and complex failure development. They were expected to follow the characteristic points given in Table 2 and show a single knee point since their intermingled layer composites showed linear stress-strain curves, but the experimental curves in Figure 16 showed a slight slope change around the intermediate elongation fibre failure strain particularly in the HMC/HSC-cSG case. Based on the experimental results, the scenario for the $\mathrm{HMC} / \mathrm{HSC}-\mathrm{cSG}$ failure development seemed to be that the HMC fibres were fractured causing whole fragmentation of the intermingled layer first, but not reaching the saturated fragmentation condition, with little dispersed delamination. The HMC fibre failure initiated crack might not be able to propagate over the 
full width or thickness of the intermingled layer as the absolute failure stress level of HMC material is much lower compared to the ultimate strength of this specimen. After that, the remaining HSC fibres seemed to fragment further around $1.93 \%$ of strain. In Figure 15(a), none of the $\mathrm{HMC} / \mathrm{EG}-\mathrm{cSG}$ composites showed a significant change of colour or pattern which would be evidence of cracks and delamination, but the $80 \%$ one is a little brighter than the other three cases. For the HMC/HSC-cSG composite with $80 \%$ of HMC content in the intermingled layer, it also showed very few cracks on the surfaces with a slightly brighter

colour than the HMC/EG-cSG composites. This suggests that many HMC fibre microfragmentations and probably dispersed delamination between the surfaces occurred.

\subsection{Application of hierarchical hybrid composites}

In the hierarchical (or multiple) hybrid composites, three main advantages can be pointed out: (i) the low elongation material increases the initial modulus of the hierarchical hybrid composites, (ii) the intermediate material delays the first failure of the low elongation material due to the fibre arrangement in the intermingled hybrid layer, and (iii) the thickness of the intermingled layer in the hierarchical hybrid can be also designed to maximise pseudoductility by multiple fragmentation and dispersed delamination. All of these lead to an increase of the area under the stress-strain curve of the hierarchical composites compared to the pure high elongation material or interlaminated hybrids, meaning that a higher amount of energy can be absorbed during tensile deformation. For example, as shown in Figure 17 (a), the hierarchical hybrid composites with $20 \% \mathrm{HMC} / 80 \% \mathrm{HSC}$ content in the intermingled layer and cSG layers showed a smoother knee point transition and higher energy absorption compared to the interlaminated hybrid composites with $100 \%$ HSC content in the middle layer and cSG layers, with the same middle layer thickness. The analytical model also supports the experimental results in Figure 17(b). 
The experimental results and fractured specimen surfaces agreed with the predictions of the Jalalvand model well, which shows that the analytical model is a promising design tool for hierarchical hybrid composites with various types of materials. Moreover, it demonstrates that hybrid composites with two types of material (low and high elongation material) arranged in a hierarchical structure offer an advantage in maximising the pseudo-ductile response. When low elongation fibres are surrounded by high elongation fibres and the intermingled layer is sandwiched between the high elongation material, Figure 18(a) A, the plateau in the stress-strain curve is longer than the one of the simple interlaminated hybrid composite, Figure 18(a) B, because of the delamination that follows the low elongation fibres fragmentation. This is because the increase of thickness ratio, $\beta$, of 'the middle layer (intermingled layer or low elongation material layer)' to 'the outer layer (high elongation material layer)' in the hierarchical configuration results in a decrease of the delamination stress according to Equation (3) [19] that determines the stress level at which delamination development occurs. Figure 18 (b) shows examples of stress-strain curves with respect to the hybrid configurations $\mathrm{A}$ and $\mathrm{B}$ based on the modified analytical model. Moreover, the intermingled arrangement in the middle layer of the hierarchical hybrid composites can maximise the hybrid effect, i.e. the delay of the first fragmentation of the low elongation material, although it was decided not to try to capture this effect in the analytical model in this paper. Therefore, the fragmentation stress can be higher than that of the interlaminated hybrid composites.

\section{Conclusion}

In this paper, different sets of intermingled hybrid composites with aligned discontinuous fibres as well as hierarchical hybrid composites - interlaminated hybrid composites with an intermingled hybrid layer sandwiched by continuous S-glass (cSG) layers, were manufactured and tested in tension. For the intermingled hybrid layers, discontinuous high 
strength carbon (HSC), high modulus carbon (HMC), and E-glass (EG) fibres were used. The experimental results of the hierarchical hybrid composites were compared with the analytical model previously proposed by the authors and further developed in this work.

Regarding the intermingled hybrid composites, $\mathrm{HMC} / \mathrm{EG}$ and $\mathrm{HMC} / \mathrm{HSC}$ showed nonlinear behaviour when the high elongation material (EG or HSC) was able to withstand the load after the low elongation material (HMC) failure, i.e. up to $60 \% \mathrm{HMC}$ fibre content. On the other hand, the intermingled hybrid composites with HSC/EG fibres did not show a plateau region in the stress-strain curves and the failure was catastrophic because the failure strain of the low elongation material (HSC) was relatively close to the failure strain of the high elongation material (EG).

The hierarchical hybrid composites failure process is greatly affected by the behaviour of the intermingled hybrid layer. When the intermingled layer had a linear stress-strain curve, the middle layer could be considered as a linear-elastic homogenised unit in the interlaminated hybrid configuration. On the other hand, when the intermingled layer had a nonlinear stressstrain behaviour the failure development could be divided into two steps; i) fragmentation of the low elongation material inside the intermingled layer and ii) fragmentation of the intermingled layer as a whole followed by dispersed delamination in some specific cases. The analytical model predicted the stress-strain curve shape taking into account the failure development described above. The HMC/HSC-cSG hierarchical hybrid composites showed two clear knee points in the stress-strain curves in agreement with the analytical solutions. This shows that this simple analytical model can predict the behaviour of hierarchical hybrid composites very well and is a promising design tool for hierarchical hybrid composites with various types of materials. The potential to increase the energy absorption was also demonstrated. Moreover, the hierarchical hybrid composites with two types of materials can be designed to maximise the pseudo-ductile strain and achieve a higher yield stress. 
Experimentally, the HiPerDiF method was confirmed as a valuable manufacturing method to create and optimise high performance hybrid composites with pseudo-ductile response.

\section{Acknowledgements and Data access statements}

This work was funded under the UK Engineering and Physical Sciences Research Council (EPSRC) Programme Grant EP/I02946X/1 on High Performance Ductile Composite Technology in collaboration with Imperial College, London. All underlying data to support the conclusions are provided within this paper.

\section{Reference}

[1] Czel G, Wisnom MR. Demonstration of pseudo-ductility in high performance glass/epoxy composites by hybridisation with thin-ply carbon prepreg. Compos Part a-Appl S 2013; 52: 23-30.

[2] Fuller JD, Jalalvand M, Wisnom MR. Combining fibre rotation and fragmentation to achieve pseudo-ductile CFRP laminates. Compos Struct 2016; 142: 155-166.

[3] Chou TW. Microstructural design of fiber composites. Cambridge solid state science series. Cambridge ; New York: Cambridge University Press, 2005.

[4] Swolfs Y, Gorbatikh L, Verpoest I. Fibre hybridisation in polymer composites: A review. Compos Part a-Appl S 2014; 67: 181-200.

[5] Fukuda H, Chou TW. Monte-Carlo Simulation of the Strength of Hybrid Composites. J Compos Mater 1982; 16(Sep): 371-385.

[6] Fukunaga H, Chou TW, Fukuda H. Probabilistic strength analyses of interlaminated hybrid composites. Compos Sci Technol 1989; 35(4): 331-345.

[7] Fukunaga H, Chou TW, Schulte K, Peters PWM. Probabilistic Initial Failure Strength of Hybrid and Non-Hybrid Laminates. J Mater Sci 1984; 19(11): 3546-3553.

[8] Dorey G, Sidey GR, Hutchings J. Impact Properties of Carbon Fiber-Kevlar-49 Fiber Hydrid Composites. Composites 1978; 9(1): 25-32.

[9] Schmeer S, Breuer UP. Carbon and metal-fiber-reinforced airframe structures. In: Friedrich K,Breuer UP, editor. Multifunctionality of Polymer Composites: Challenges and New Solutions. Elsevier, 2015.

[10] Richter H. Single fibre and hybrid composites with aligned discontinuous fibres in polymer matrix. In: Proceedings of ICCM-3. Paris, France, 1980.

[11] Edwards H, Evans NP. A method for the production of high quality aligned short fibre mats and their composites. in 3rd International Conference on Composite Materials. Paris, France, 1980.

[12] Parratt NJ, Potter KD. Mechanical behaviour of intimately-mixed hybrid composites. In: Proceedings of International Conference on Composite Materials. Paris, France, 1980. p. 313-323.

[13] Potter KD. Deformation mechanisms of fibre reinforcements and their influence on the fabrication of complex structural parts In: Proceedings of ICCM-3. Paris, France 1980.

[14] Yu H, Longana ML, Jalalvand M, Wisnom MR, Potter KD. Pseudo-ductility in intermingled carbon/glass hybrid composites with highly aligned discontinuous fibres. Composites: Part A 2015; 73: 35-44. 
[15] Longana ML, Yu H, Potter KD. Aligned virgin and recycled short carbon fibre hybrid composites. in 20th International Conference on Composite Materials. Copenhagen, 2015.

[16] Yu H, Potter KD, Wisnom MR. A novel manufacturing method for aligned discontinuous fibre composites (High Performance-Discontinuous Fibre method). Compos Part a-Appl S 2014; 65: 175-185.

[17] Qian H, Greenhalgh ES, Shaffer MSP, Bismarck A. Carbon nanotube-based hierarchical composites: a review. J Mater Chem 2010; 20(23): 4751-4762.

[18] Pimenta S, Pinho ST. Hierarchical scaling law for the strength of composite fibre bundles. J Mech Phys Solids 2013; 61(6): 1337-1356.

[19] Jalalvand M, Czel G, Wisnom MR. Damage analysis of pseudo-ductile thin-ply UD hybrid composites-a new analytical method. Compos Part a-Appl S 2015; 69: 83-93.

[20] Yu H, Longana ML, Grail G, Pimental S, Robinson P, Wisnom MR, Potter KD. Aligned short fibre composites with nonlinear behaviour. In: Proceedings of ICCM20. Copenhagen, Denmark, 2015.

[21] Wisnom MR, Czel G, Swolfs Y, Jalalvand M, Gorbatikh L, Verpoest I. Hybrid effects in thin ply carbon/glass unidirectional laminates: Accurate experimental determination and prediction. Compos Part a-Appl S 2016; 88: 131-139.

[22] ASTM international. D3039/D3039M - 14 Standard Test Method for Tensile Properties of Polymer Matrix Composite Materials.

[23] Zweben C. Tensile-Strength of Hybrid Composites. Astronaut Aeronaut 1977; 15(10): B24-B24.

[24] Marom G, Fischer S, Tuler FR, Wagner HD. Hybrid Effects in Composites Conditions for Positive or Negative Effects Versus Rule-of-Mixtures Behavior. J Mater Sci 1978; 13(7): 1419-1426.

[25] Swolfs Y, Verpoest I, Gorbatikh L. Maximising the hybrid effect in unidirectional hybrid composites. Mater Design 2016; 93: 39-45.

[26] Swolfs Y, Gorbatikh L, Verpoest I. Stress concentrations in hybrid unidirectional fibre-reinforced composites with random fibre packings. Compos Sci Technol 2013; 85: $10-16$.

[27] Heuvel PWJ, Goutianos S, Young RJ, Peijs T. Failure phenomena in fibre-reinforced composites. Part 6: a finite element study of stress concentrations in unidirectional carbon fibre-reinforced epoxy composites. Compos Sci Technol 2004; 64(5): 645-656.

\section{Figure captions}

Figure 1. Schematic of intermingled hybrid composite and hierarchical interlaminated hybrid composite specimens.

Figure 2. (a) The procedure for finding the hierarchical hybrid's damage process based on the order of required stresses for each damage mode, (b) Stress-strain response of possible damage scenarios for hierarchical hybrid composites with having linear-elastic behaviour of intermingled hybrids. (See Table 1.)

Figure 3. (a) The procedure for finding the hierarchical hybrid's damage process based on the order of required stresses for each damage mode, (b) Stress-strain response of possible damage scenarios for hierarchical hybrid composites with having nonlinear behaviour of intermingled hybrids. (see Table 2.)

Figure 4. Single unit of fibre orientation head in HiPerDiF method [16]. 
Figure 5. Schematic of lab-scale aligned discontinuous fibre prepregging rig [20].

Figure 6. Specimen dimension for tensile test reffered to ASTM3039.

Figure 7. Representative stress-strain curves for (a) HSC/EG specimens as a function of the HSC fibre content, (b) HMC/EG and (c) HMC/HSC specimens as a function of the HMC content.

Figure 8. Representative stress-strain curves of hierarchical hybrid specimens with continuous S-glass (cSG) and intermingled discontinuous layers of (a) HSC/EG as a function of the HSC content*, (b) HMC/EG and (c) HMC/HSC as a function of the HMC content*. (Colour codes provided in electronic version of the paper.) (* The content $\%$ is in the intermingled layer.)

Figure 9. Yield stress and Pseudo-ductile strain of (a) HMC/EG intermingled hybrids and (b) $\mathrm{HMC} / \mathrm{HSC}$ intermingled hybrids as a function of the low elongation fibres (HMC) content.

Figure 10. Failure stress of intermingled hybrids as a function of the low elongation fibres content.

Figure 11. (a) The measured failure strain of the low elongation material (HMC) at the knee point of $\mathrm{HMC} / \mathrm{EG}$ and $\mathrm{HMC} / \mathrm{HSC}$ composites as a function of the $\mathrm{HMC}$ content ratio, (b) Stress-strain curves of $\mathrm{HMC} / \mathrm{EG}$ and $\mathrm{HMC} / \mathrm{HSC}$ composites at 20, 40, 60\% $\mathrm{HMC}$ content.

Figure 12. Experimental and predicted stress-strain curves of HSC/EG-cSG hierarchical hybrid composites. (See Table 1.)

Figure 13. Top surfaces of tested HSC/EG-cSG hybrid specimens as the low elongation fibre content (HSC) in the intermingled layer. (*Bright yellow colour denotes delaminated area between the intermingled layer and S-glass layer.)

Figure 14. Experimental and predicted stress-strain curves of (a) HMC/EG -cSG and (b) HMC/HSC-cSG hierarchical hybrid composites at 20, 40, 60\% HMC fibre content in the intermingled layer. (see Table 2.)

Figure 15. Top surfaces of tested (a) HSC/EG-cSG and (b) HMC/HSC-cSG hybrid specimens as the low elongation fibre content (HMC) in the intermingled layer.

Figure 16. Experimental and predicted stress-strain curves of hierarchical hybrid composites at $80 \%$ of HMC fibre content in the intermingled layer according to the analytical model for linear (L) behaviour of intermingled layer.

Figure 17. Comparison hierarchical hybrid composite (20\% HMC/80\% HSC-cSG) with interlaminated hybrid composite (100\% HSC-cSG): (a) Experimental and (b) Analytical model predicted stress-strain curves.

Figure 18. (a) Schematic diagram for comparison between hierarchical hybrids and interlaminated hybrids with two types of fibre, (b) their stress-strain curves.

\section{Table captions}

Table 1 Coordinates of characteristic points of different processes on stress-strain graph for hierarchical hybrid composites having a Linear behaviour of the intermingled layer 
Table 2 Coordinates of characteristics points of different processes on stress-strain graph for hierarchical hybrid composites having a Nonlinear behaviour of the intermingled layer

Table 3 Constant machine parameters

Table 4 Fibres properties

Table 5 Test results summary for HSC/EG intermingled hybrid composites

Table 6 Test results summary for HMC/EG intermingled hybrid composites

Table 7 Test results summary for HMC/HSC intermingled hybrid composites

Table 8 Test results summary for HSC/EG intermingled hybrid with continuous SG composites (HSC/EG-cSG)

Table 9 Test results summary for HMC/EG intermingled hybrid with continuous SG composites (HMC/EG-cSG)

Table 10 Test results summary for HMC/HSC intermingled hybrid with continuous SG composites (HMC/HSC-cSG)

Table 11 Continuous S-glass composite properties 
IE: Intermediate Elongation Material

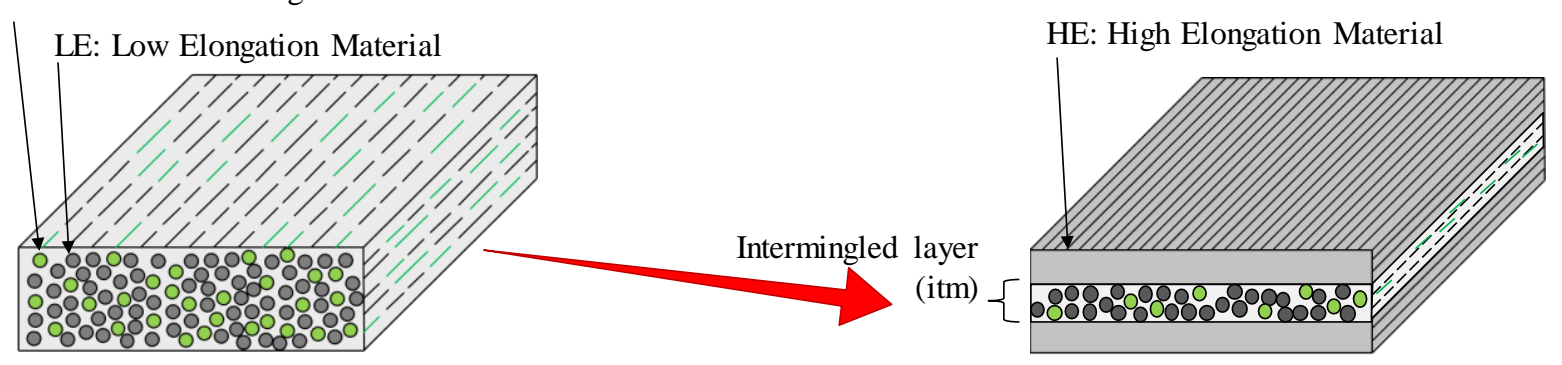

Figure 1. Schematic of intermingled hybrid composite and hierarchical interlaminated hybrid composite specimens.

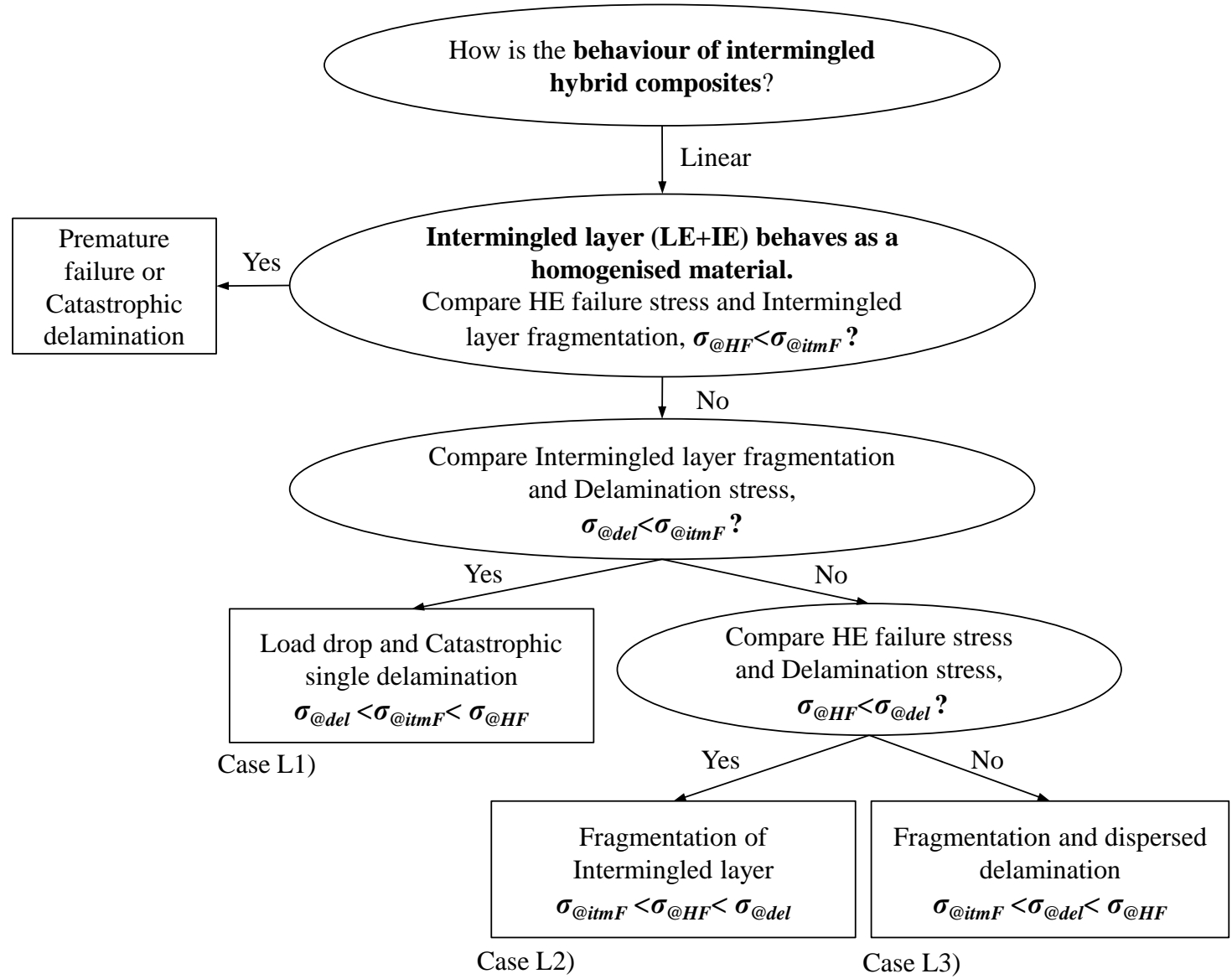

(a) 

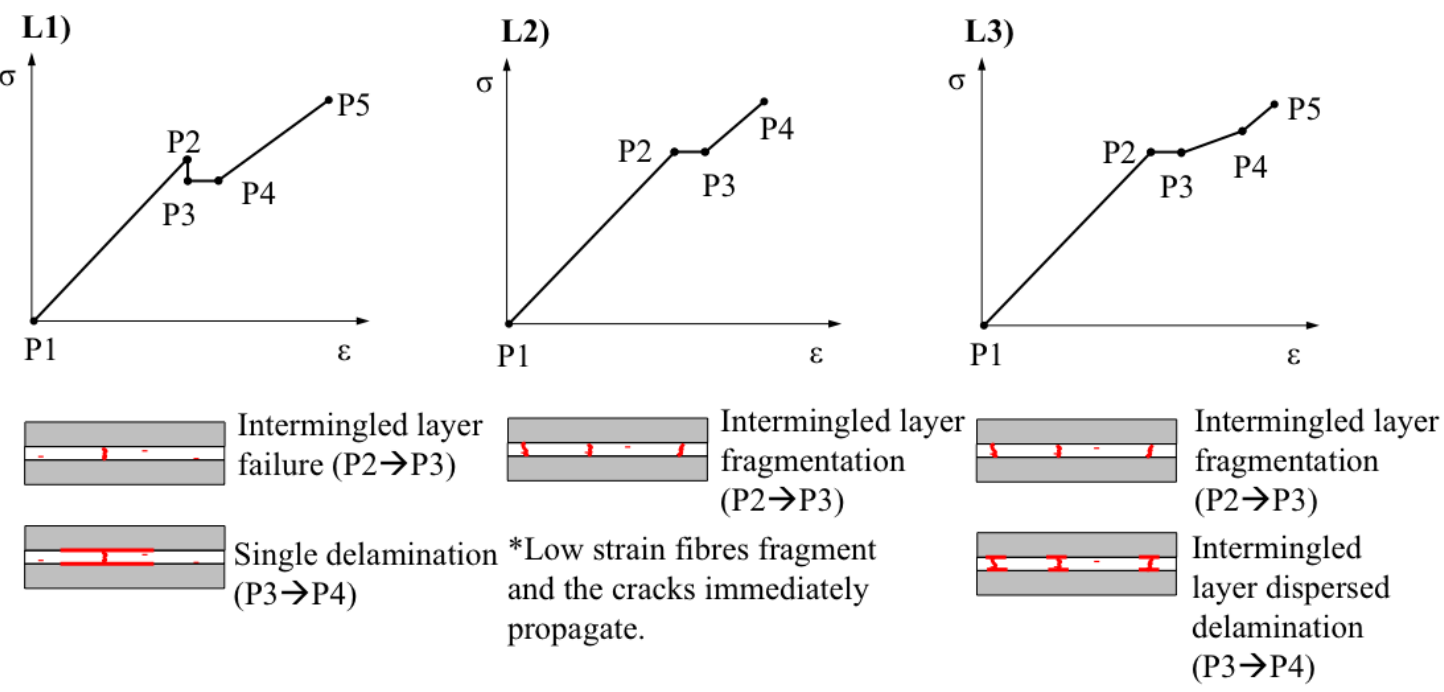

(b)

Figure 2. (a) The procedure for finding the hierarchical hybrid's damage process based on the order of required stresses for each damage mode, (b) Stress-strain response of possible damage scenarios for hierarchical hybrid composites with having linear-elastic behaviour of intermingled hybrids. (See Table 1.) 


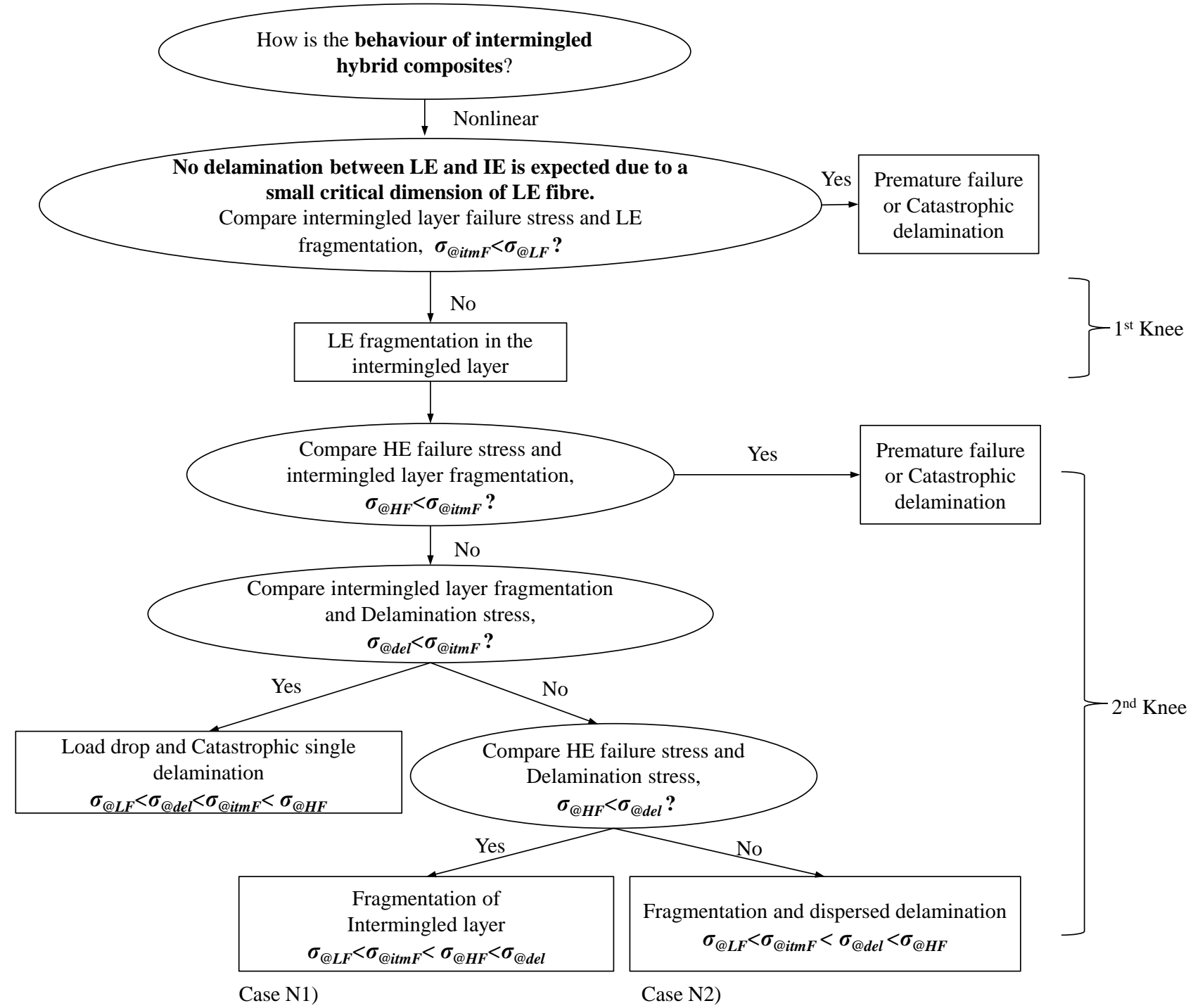

(a) 
N1)

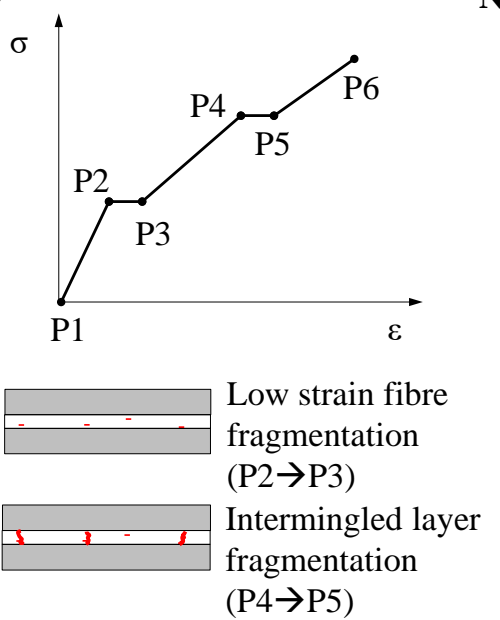

N2)
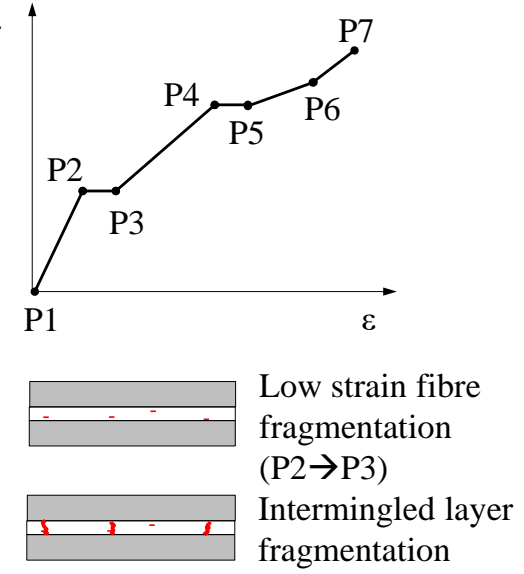

(P4 $\rightarrow$ P5)

Intermingled layer dispersed delamination

$(\mathrm{P} 5 \rightarrow \mathrm{P} 6)$

(b)

Figure 3. (a) The procedure for finding the hierarchical hybrid's damage process based on the order of required stresses for each damage mode, (b) Stress-strain response of possible damage scenarios for hierarchical hybrid composites with having nonlinear behaviour of intermingled hybrids. (see Table 2.)

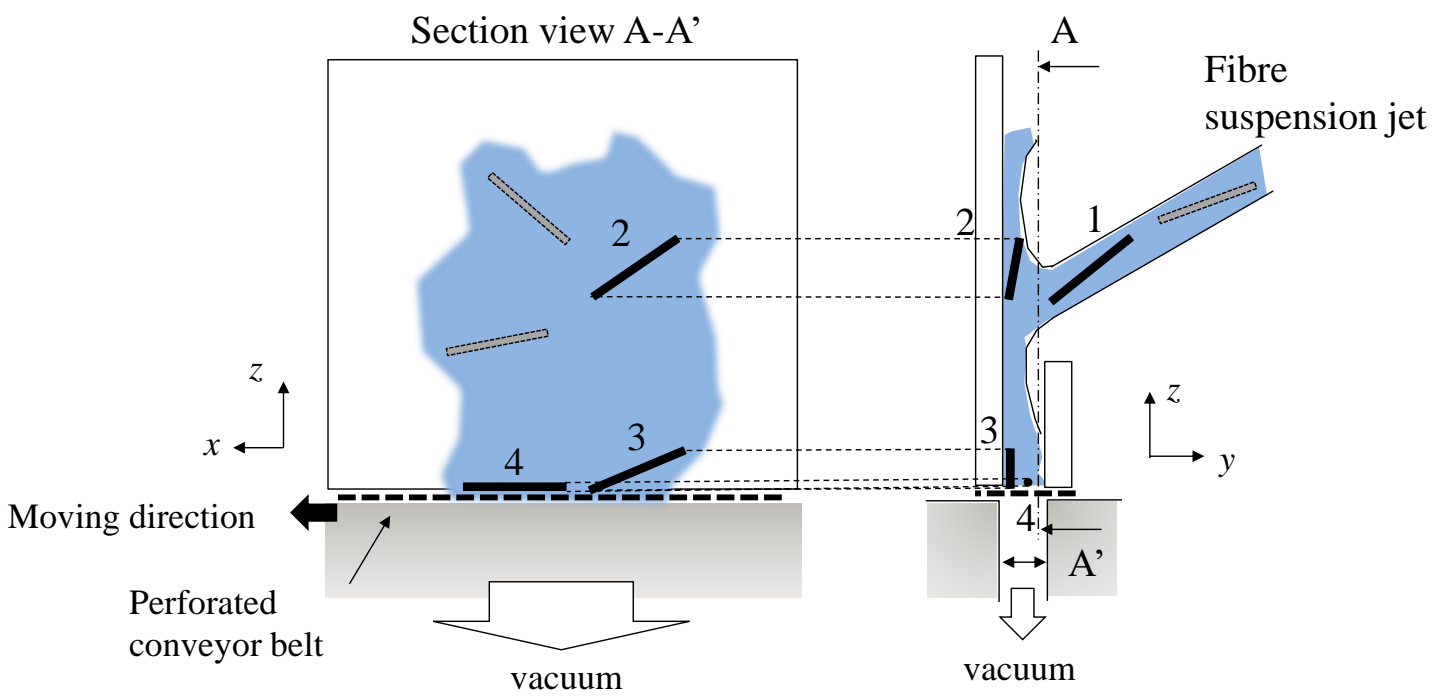

Water suction plate with a slit (slit width = gap width between parallel plates)

Figure 4. Single unit of fibre orientation head in HiPerDiF method [16]. 


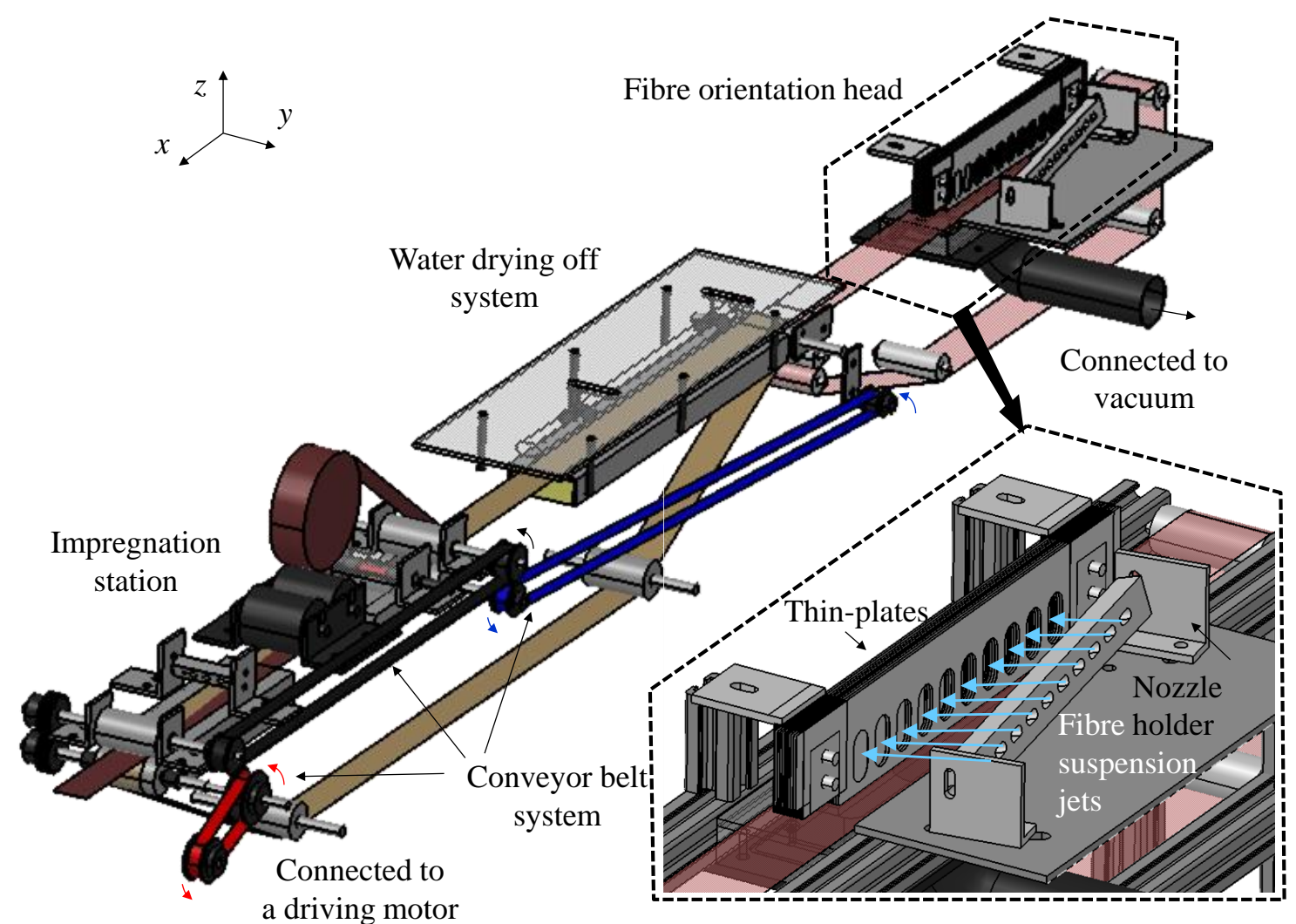

Figure 5. Schematic of lab-scale aligned discontinuous fibre prepregging rig [20].

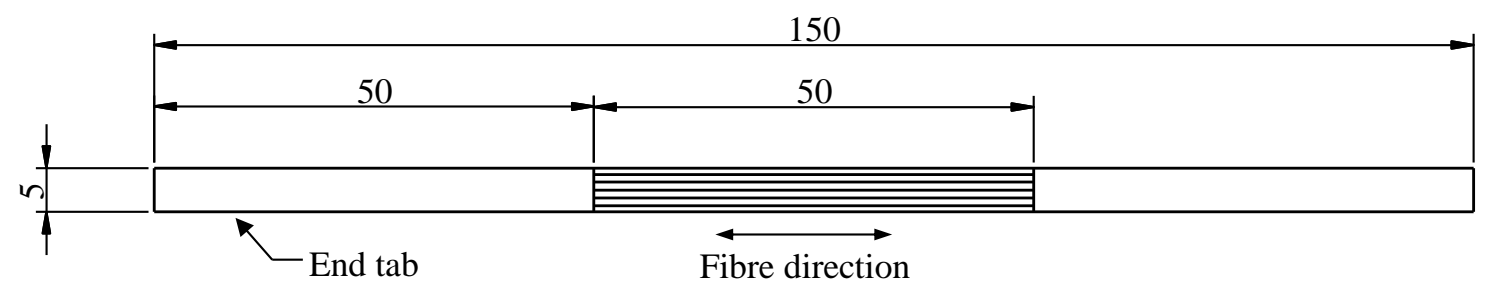

Figure 6. Specimen dimension for tensile test referred to ASTM D3039. 


\section{HSC/EG}

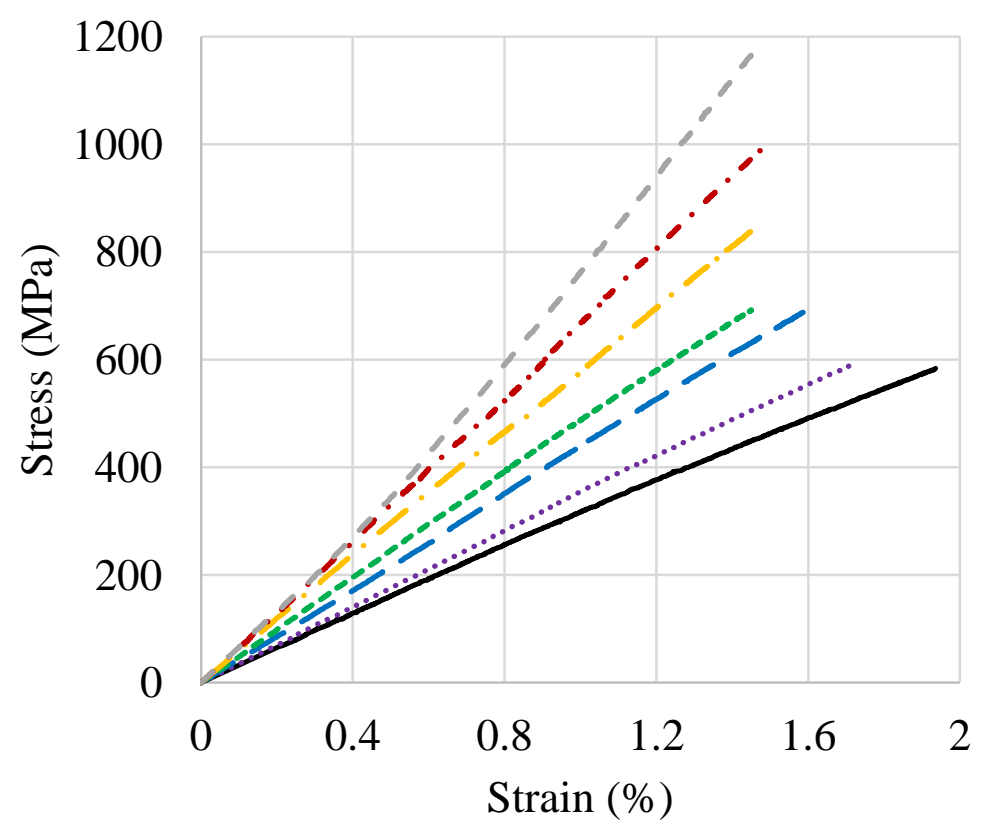

- HSC 0\%

........ HSC $20 \%$

- - HSC $40 \%$

---..HSC $50 \%$

- - HSC $60 \%$

-. - HSC $80 \%$

- - $\mathrm{HSC} 100 \%$

(a)

\section{HMC/EG}

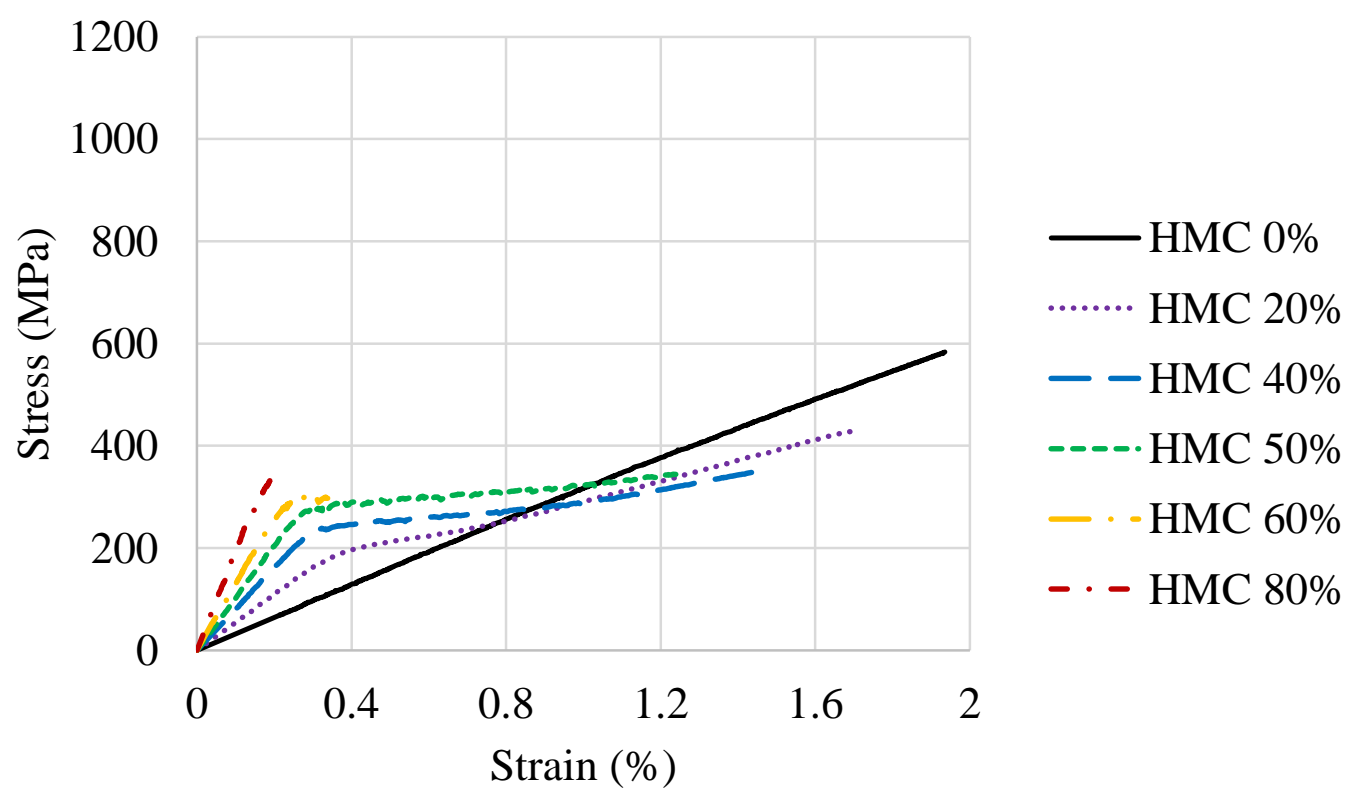

(b) 


\section{$\mathrm{HMC} / \mathrm{HSC}$}

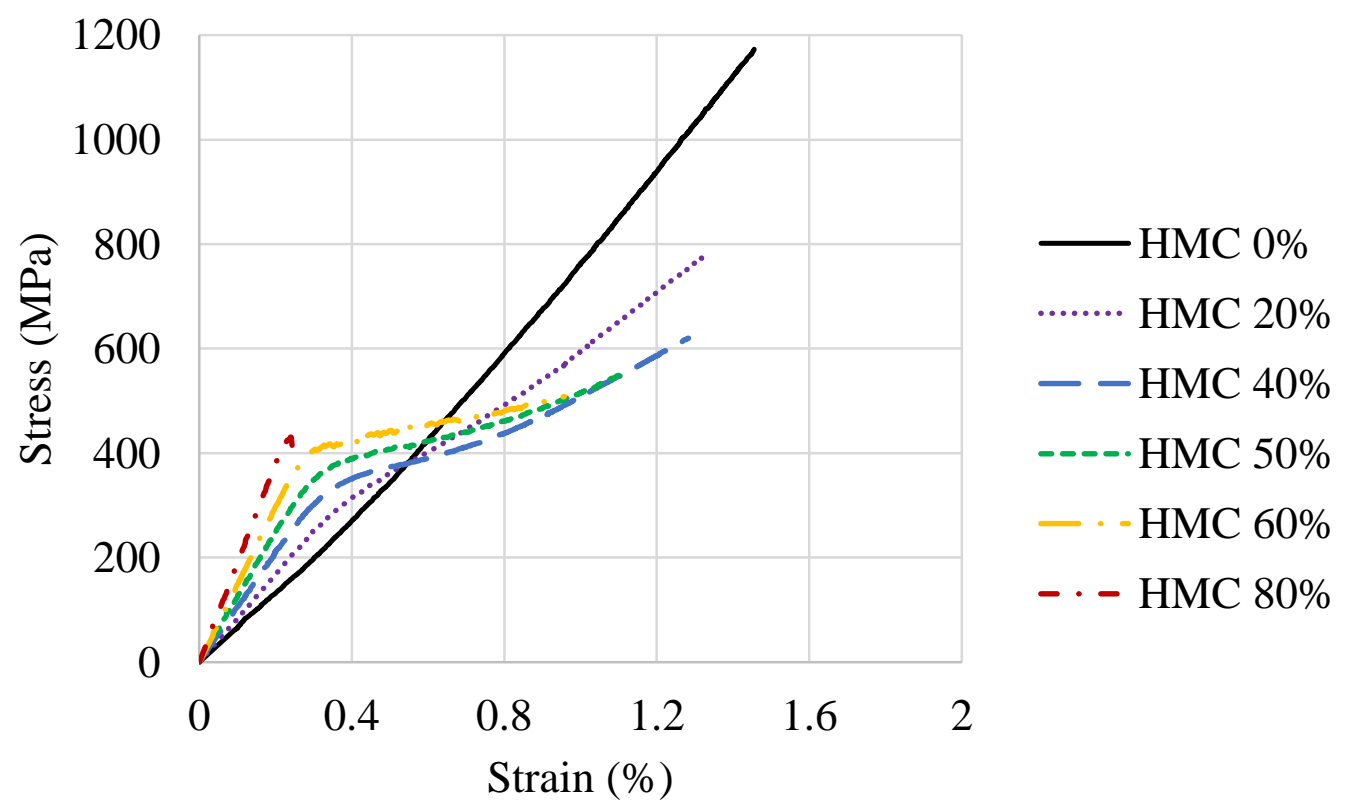

(c)

Figure 7. Representative stress-strain curves for (a) HSC/EG specimens as a function of the HSC fibre content, (b) HMC/EG and (c) HMC/HSC specimens as a function of the HMC content.

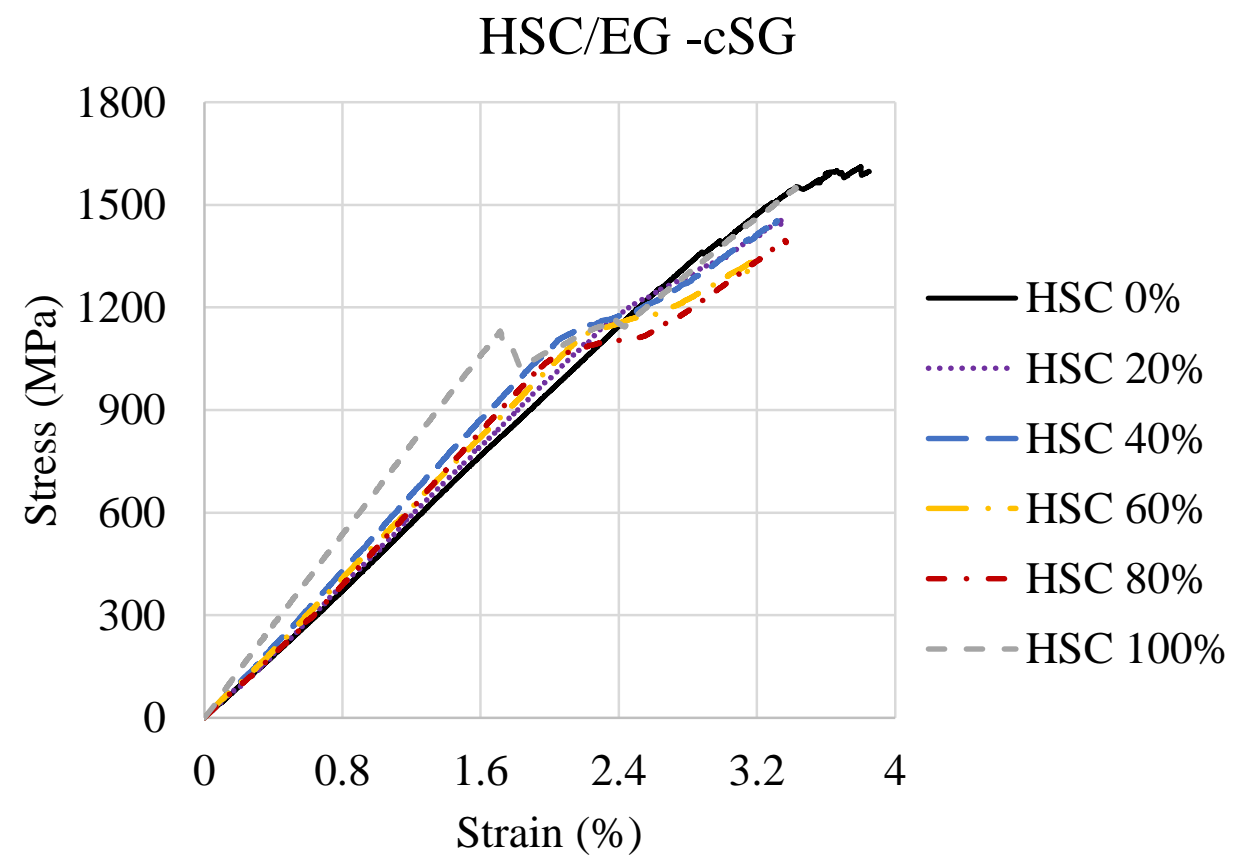

(a) - need to correct 


\section{$\mathrm{HMC} / \mathrm{EG}-\mathrm{cSG}$}

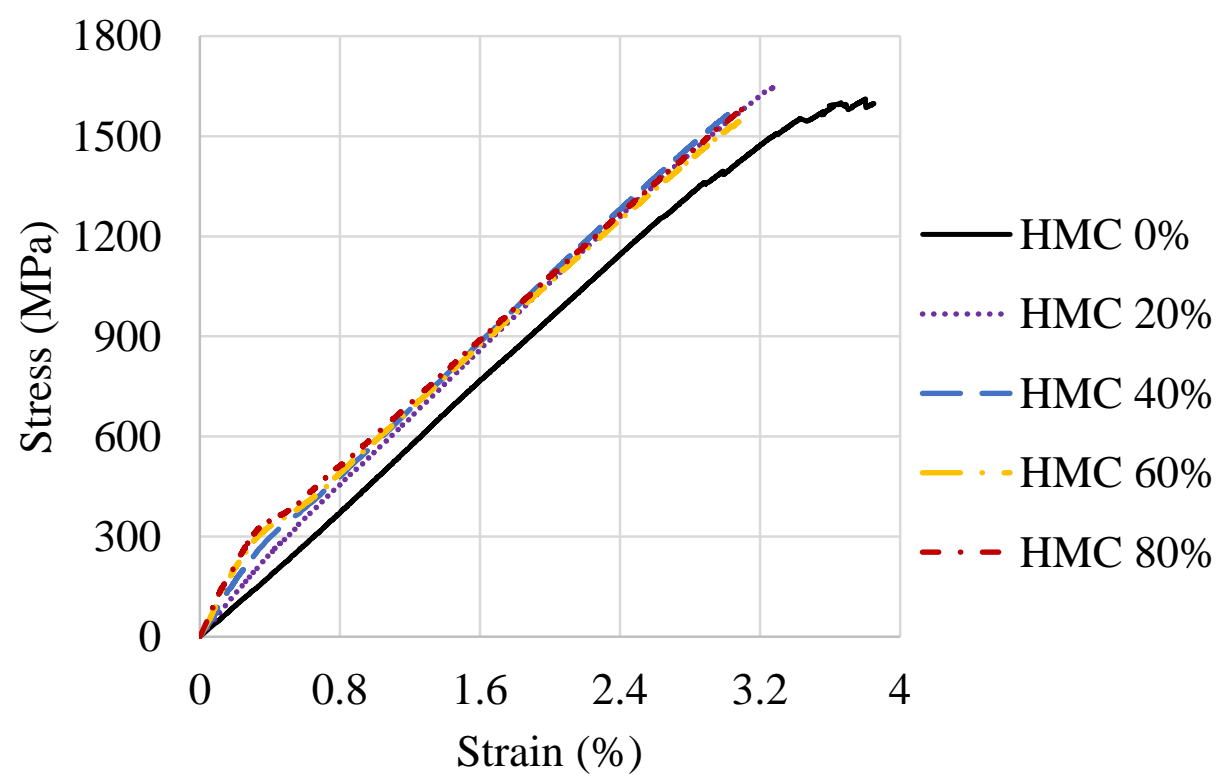

(b)

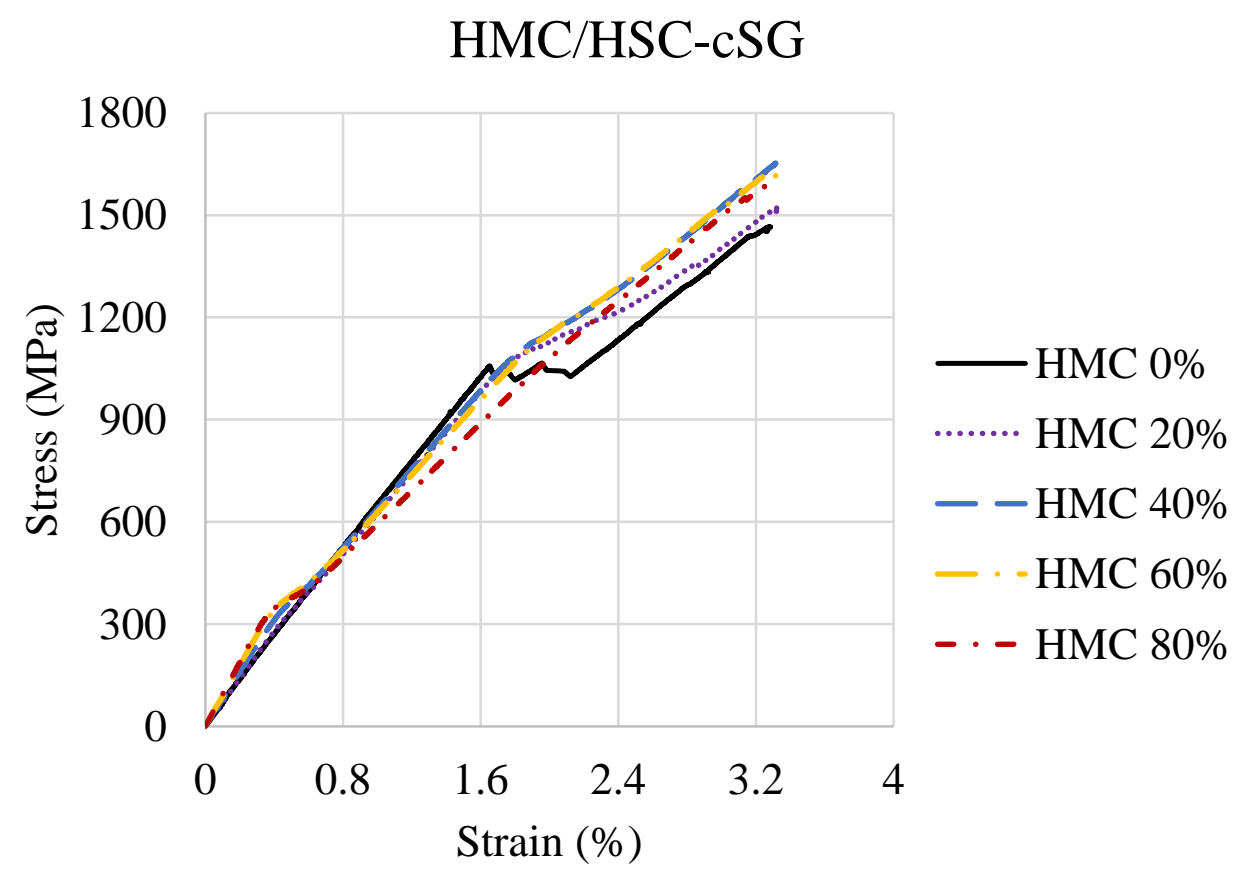

(c)

Figure 8. Representative stress-strain curves of hierarchical hybrid specimens with continuous S-glass (cSG) and intermingled discontinuous layers of (a) HSC/EG as a function of the HSC content*, (b) HMC/EG and (c) HMC/HSC as a function of the HMC content*. (Colour codes provided in electronic version of the paper.)

(* The content $\%$ is in the intermingled layer.) 


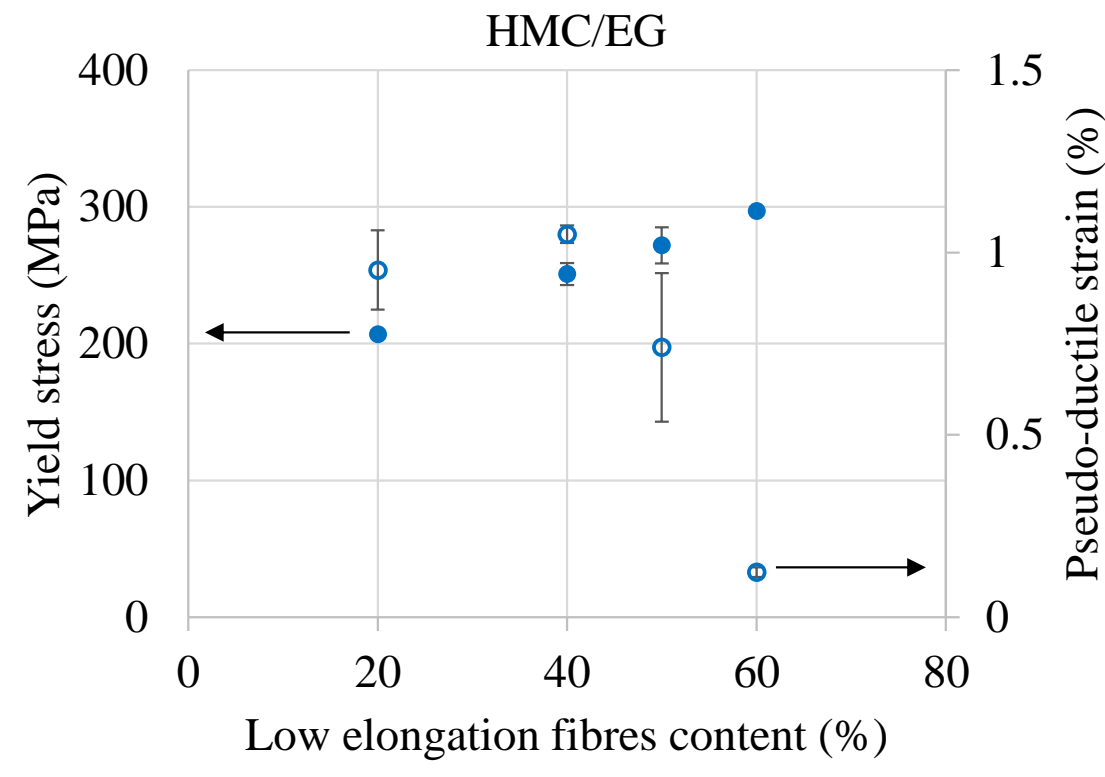

(a)

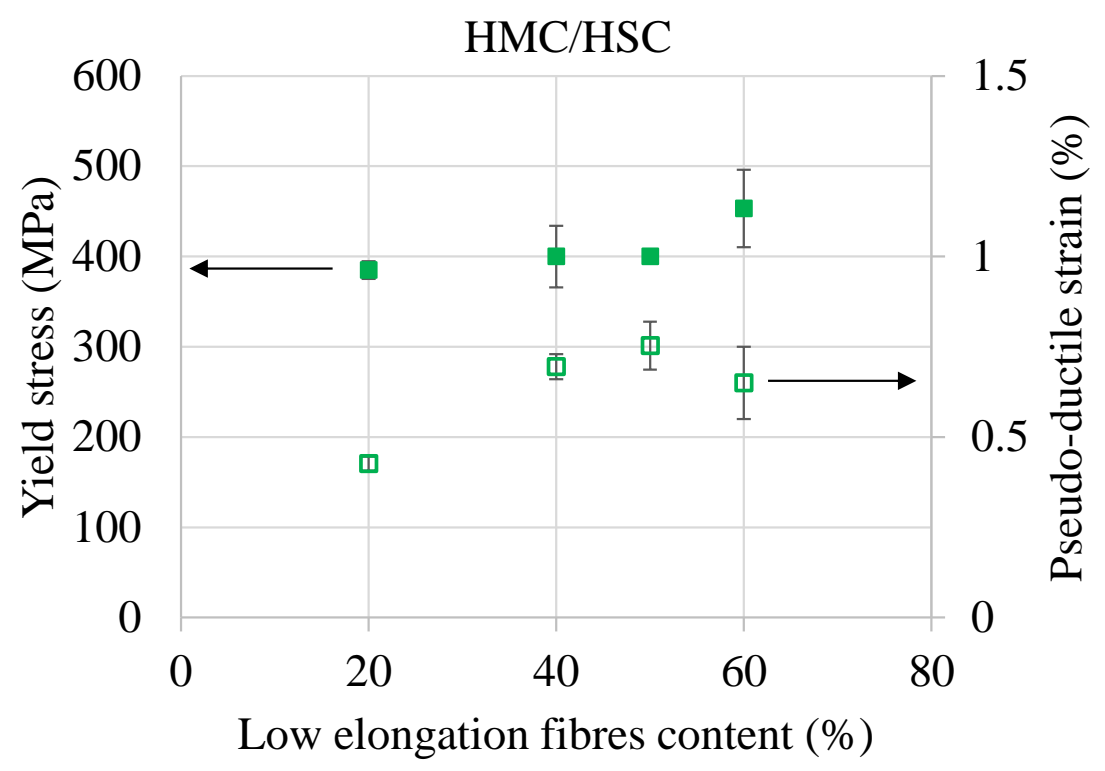

(b)

Figure 9. Yield stress and Pseudo-ductile strain of (a) HMC/EG intermingled hybrids and (b) $\mathrm{HMC} / \mathrm{HSC}$ intermingled hybrids as a function of the low elongation fibres (HMC) content. 


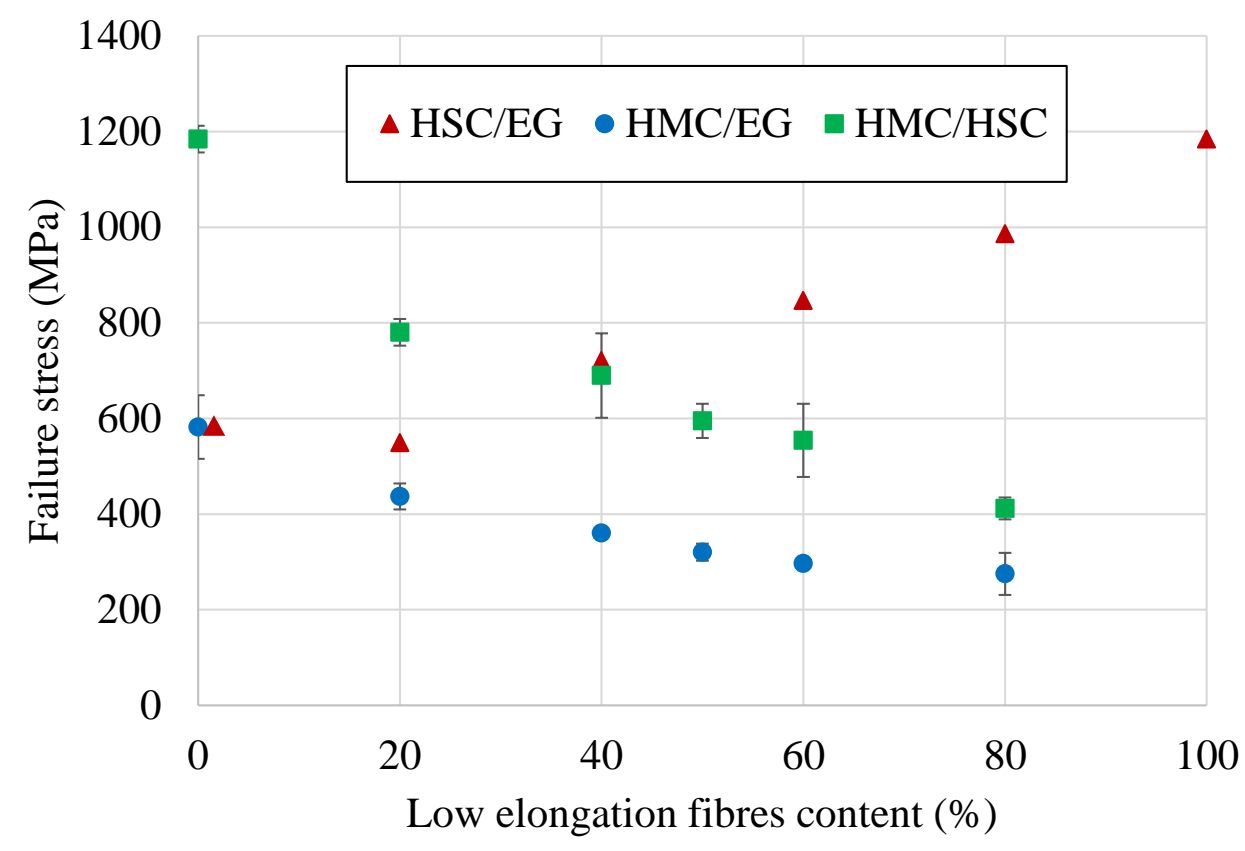

Figure 10. Failure stress of intermingled hybrids as a function of the low elongation fibres content. - Bilinear rule of mixtures line will be added. 


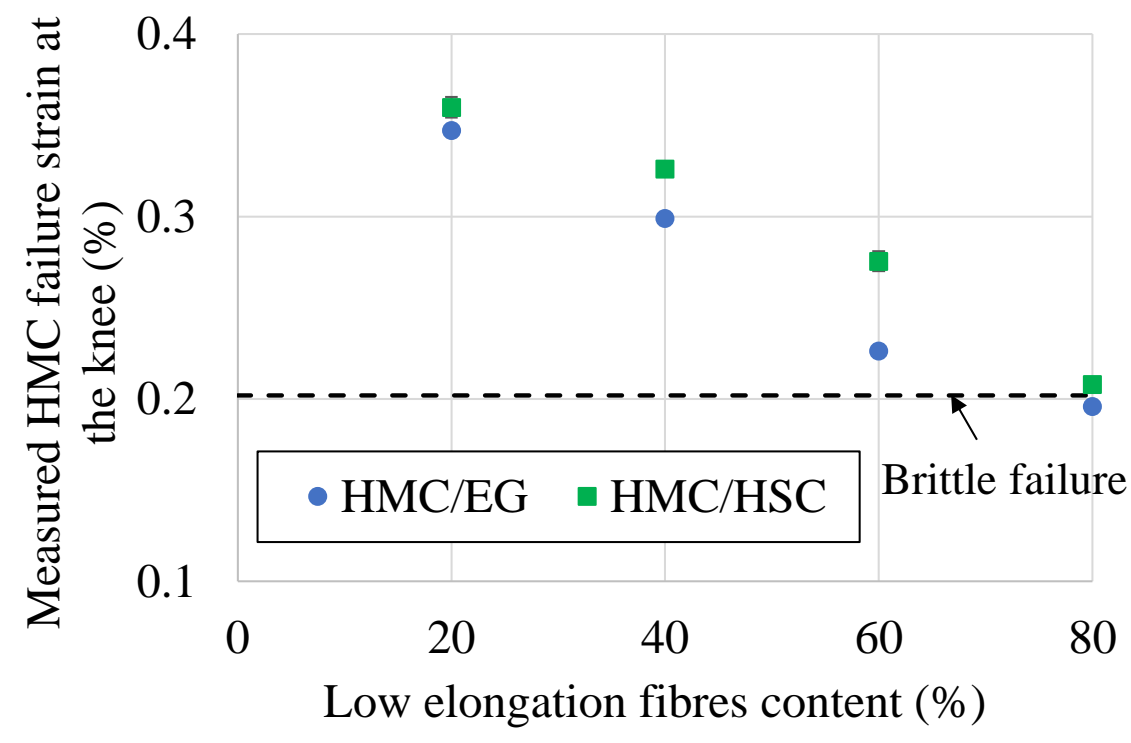

(a)

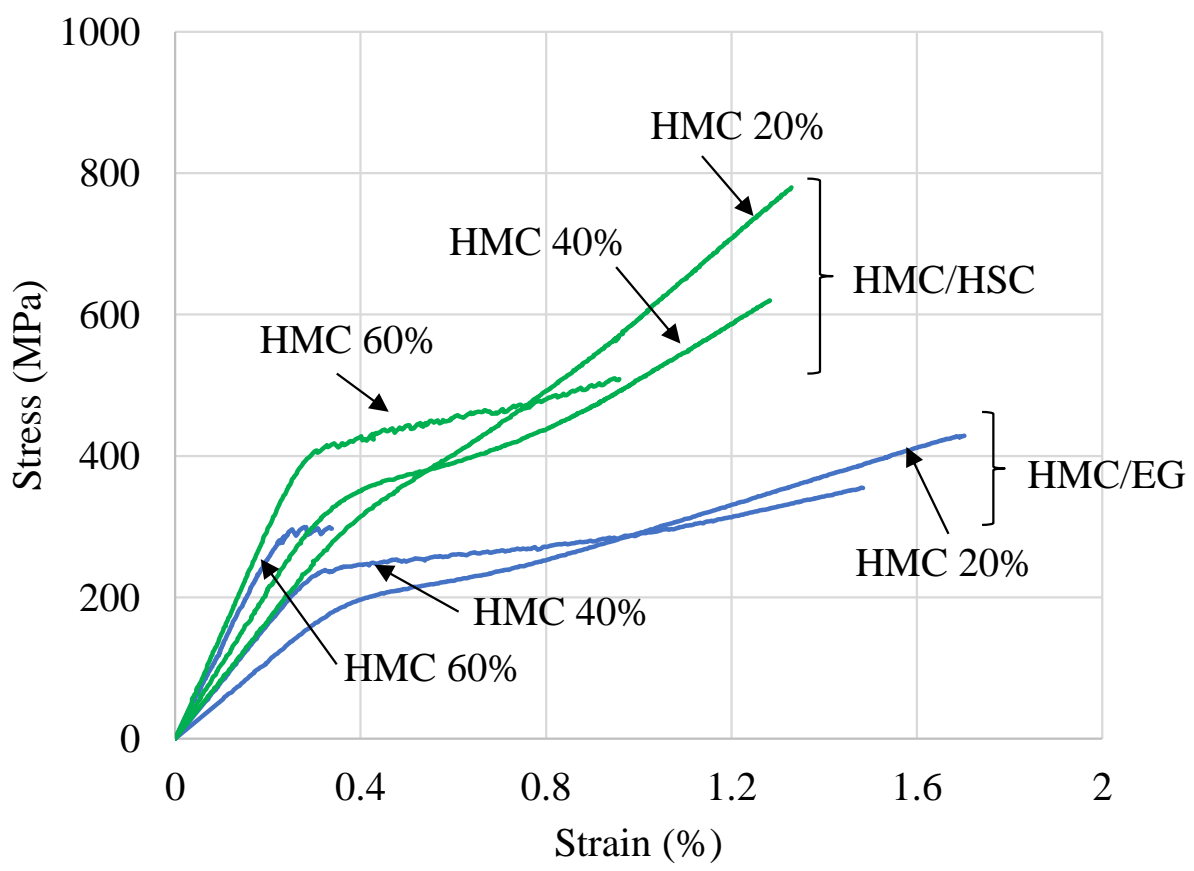

(b)

Figure 11. (a) The measured failure strain of the low elongation material (HMC) at the knee point of $\mathrm{HMC} / \mathrm{EG}$ and $\mathrm{HMC} / \mathrm{HSC}$ composites as a function of the HMC content ratio, (b) Stress-strain curves of HMC/EG and HMC/HSC composites at 20, 40, 60\% HMC content. 

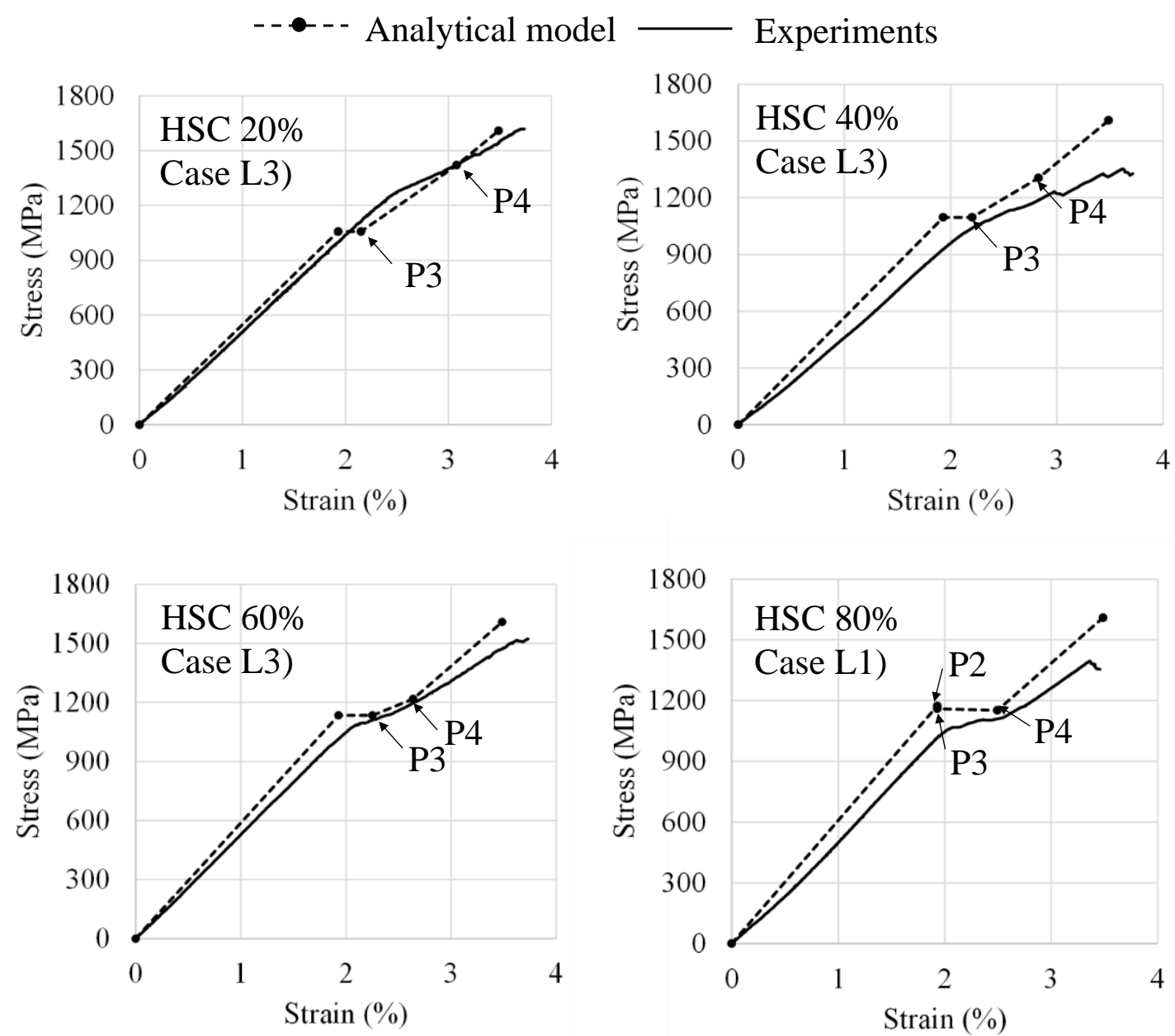

Figure 12. Experimental and predicted stress-strain curves of HSC/EG-cSG hierarchical hybrid composites. (See Table 1.)

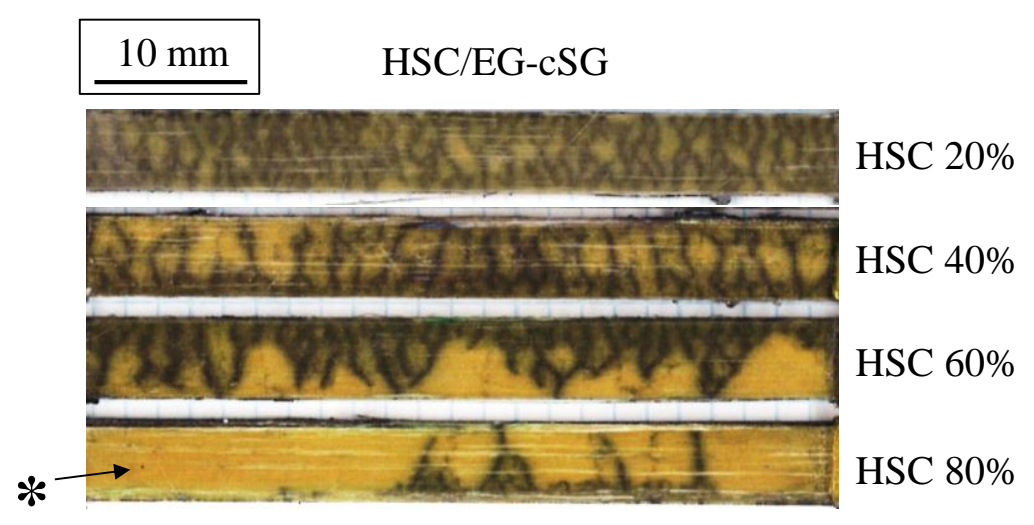

Figure 13. Top surfaces of tested HSC/EG-cSG hybrid specimens as the low elongation fibre content (HSC) in the intermingled layer.

(*Bright yellow colour denotes delaminated area between the intermingled layer and S-glass layer.) 
-- --- Analytical model —— Experiments
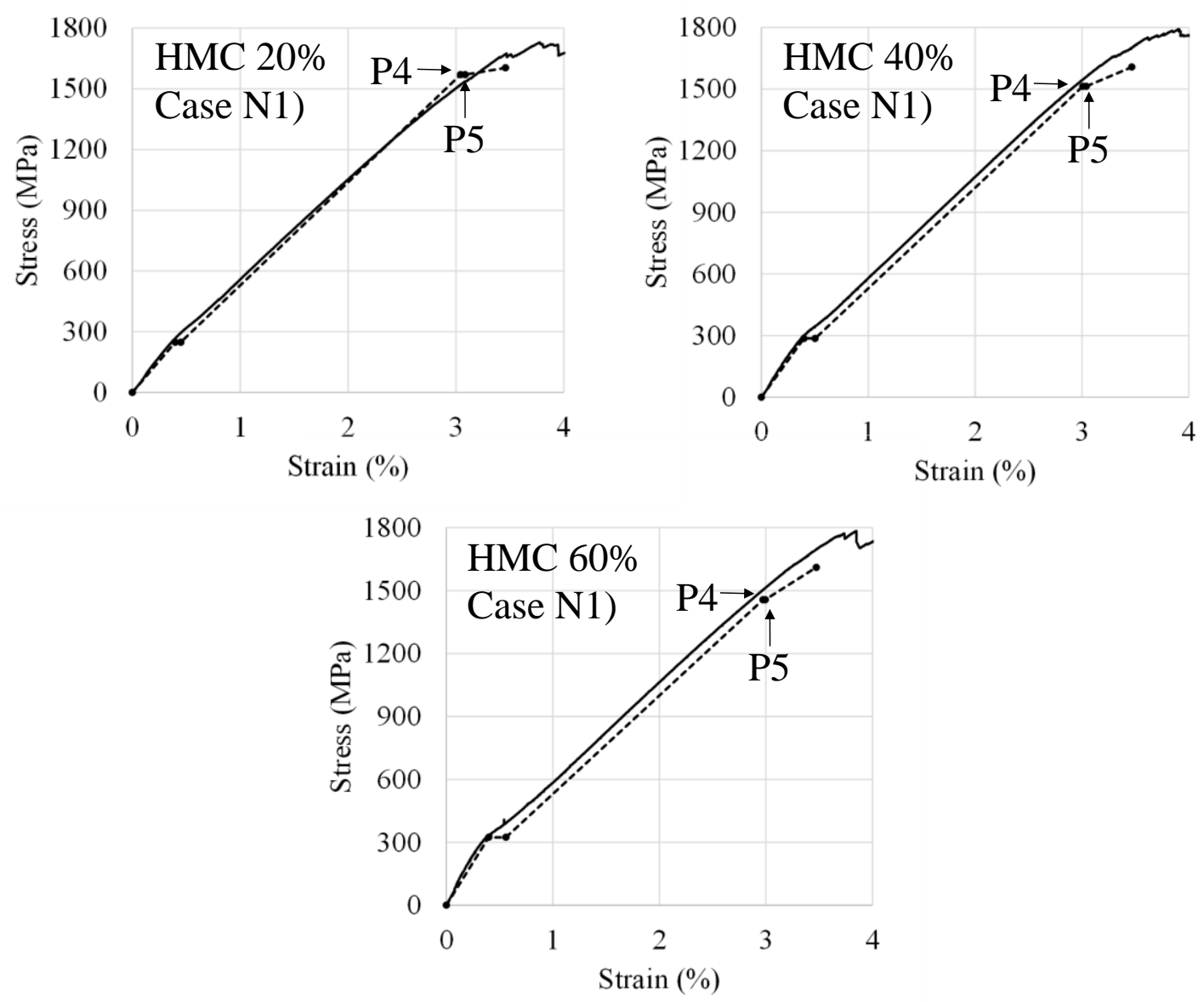

(a) 


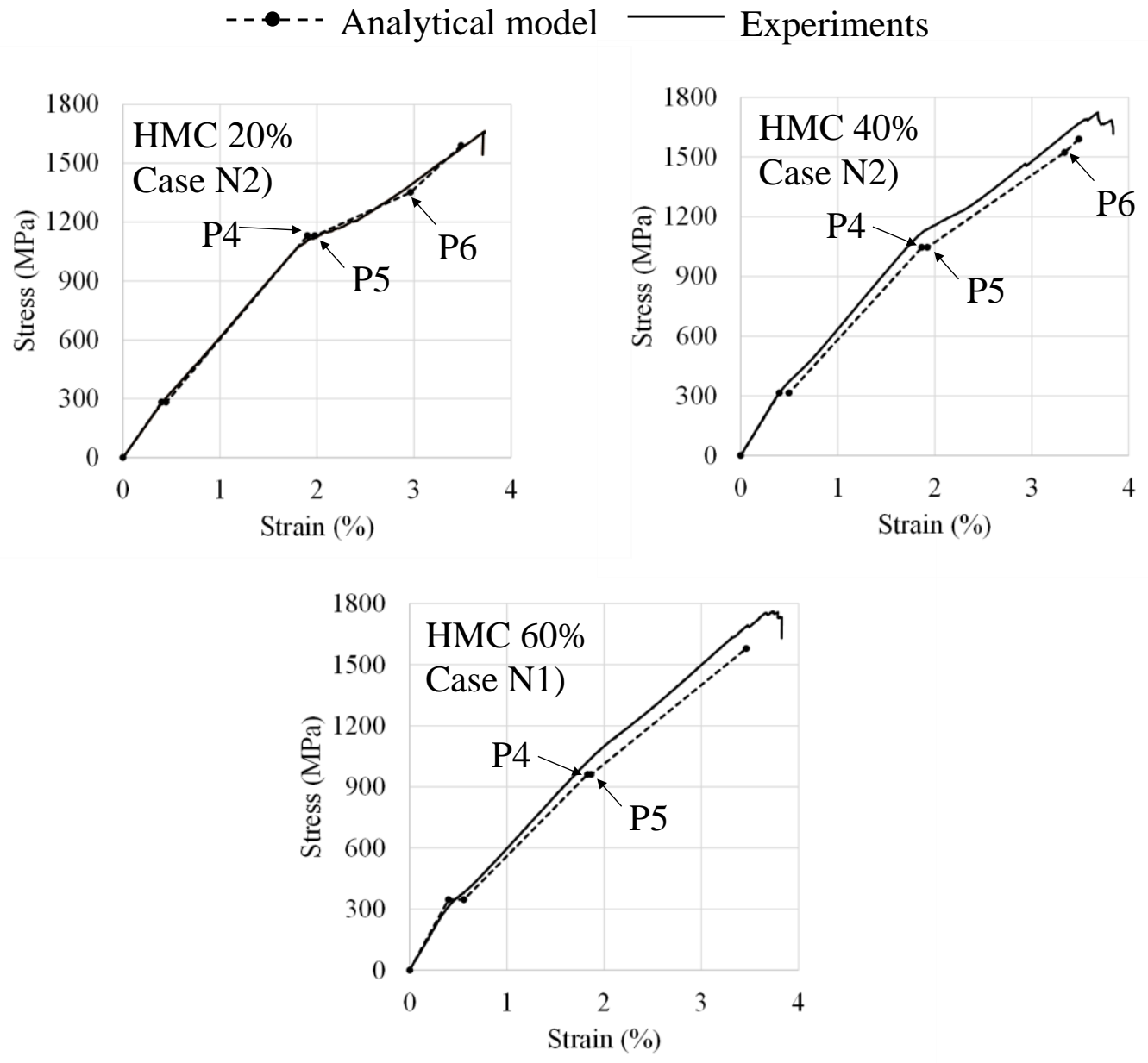

(b)

Figure 14. Experimental and predicted stress-strain curves of (a) HMC/EG -cSG and (b) HMC/HSC-cSG hierarchical hybrid composites at 20, 40, 60\% HMC fibre content in the intermingled layer. (see Table 2.)

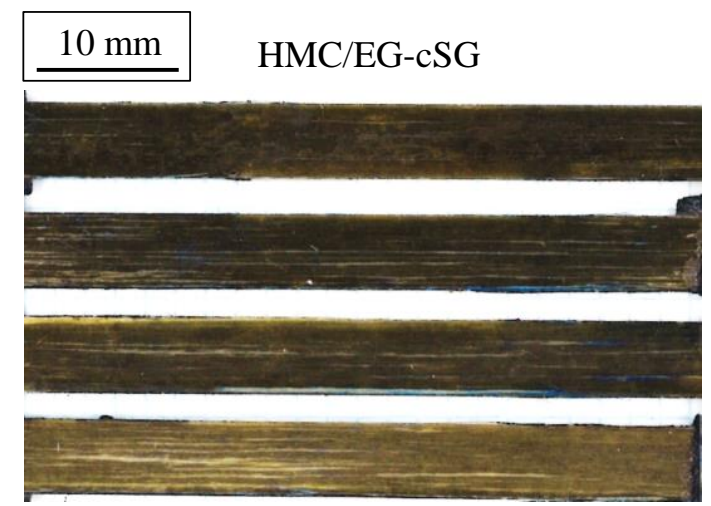

(a)

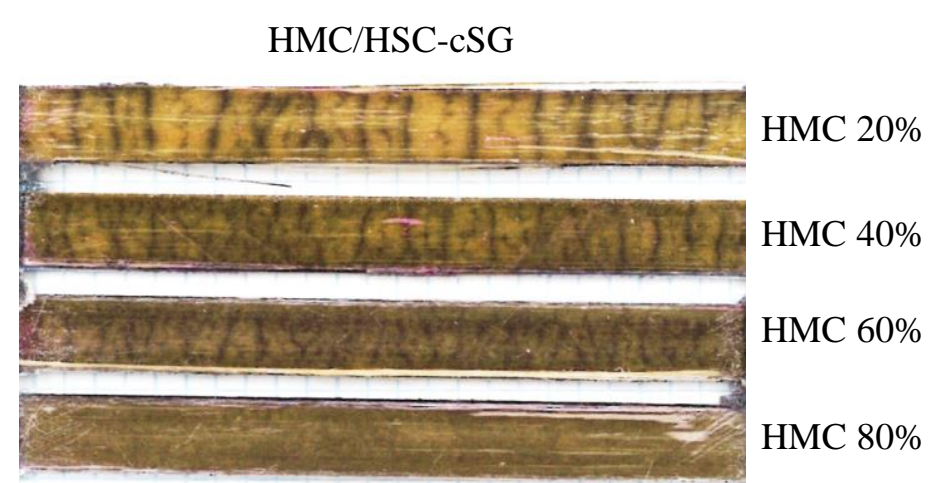

(b)

Figure 15. Top surfaces of tested (a) HSC/EG-cSG and (b) HMC/HSC-cSG hybrid specimens as the low elongation fibre content (HMC) in the intermingled layer. 
………... Analytical model (L)

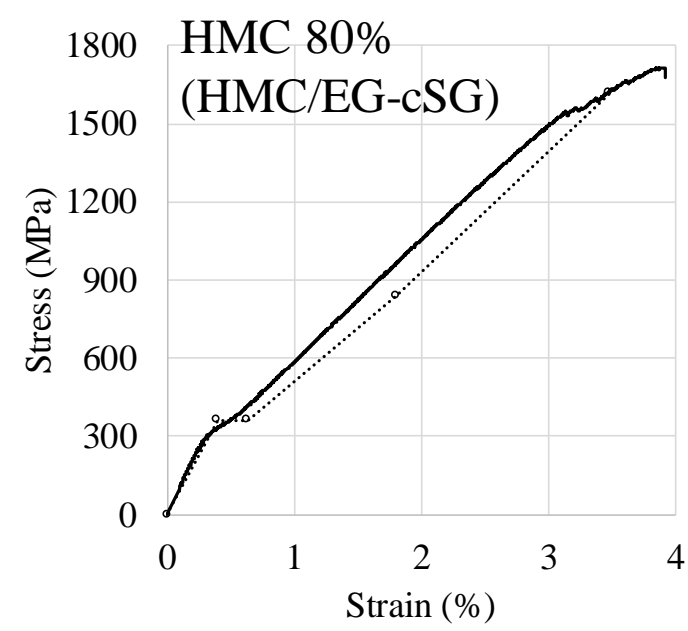

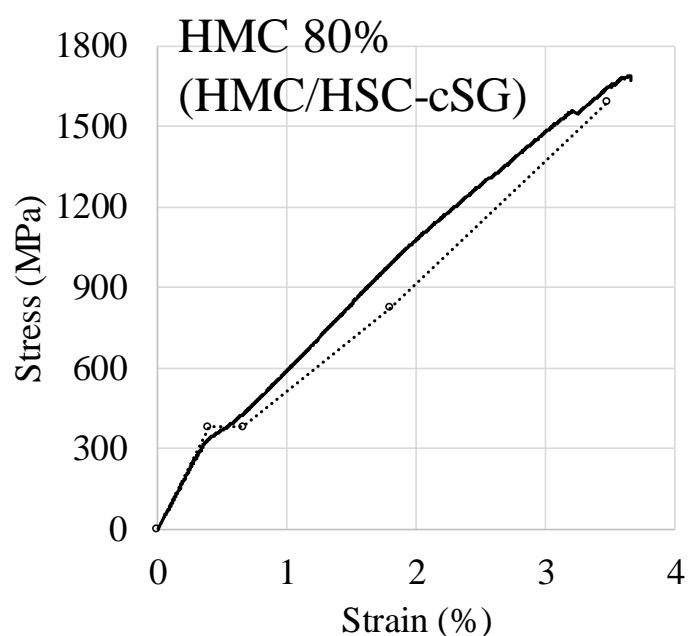

Figure 16. Experimental and predicted stress-strain curves of hierarchical hybrid composites at $80 \%$ of HMC fibre content in the intermingled layer according to the analytical model for linear (L) behaviour of intermingled layer.

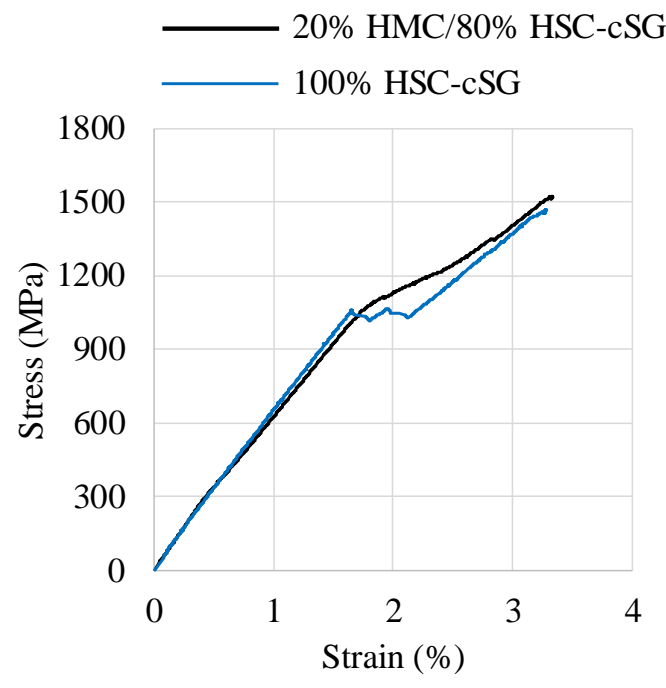

(a)

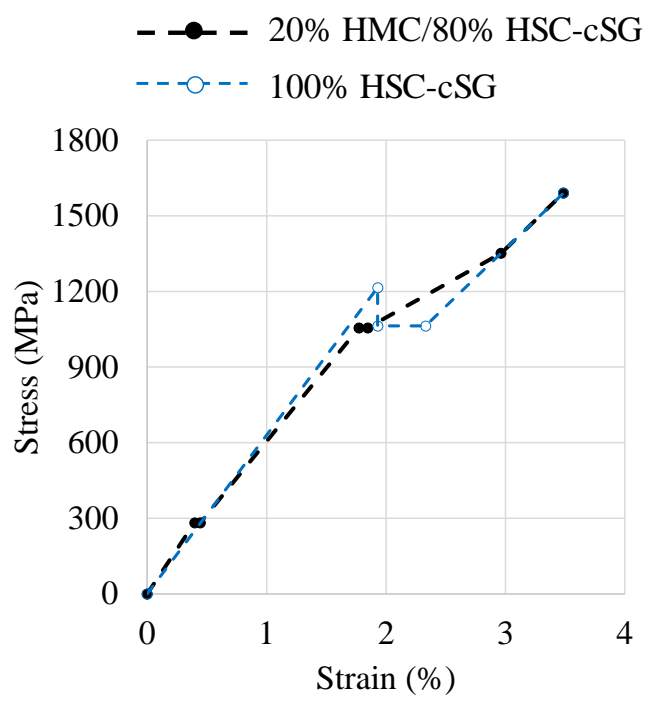

(b)

Figure 17. Comparison hierarchical hybrid composite (20\% HMC/80\% HSC-cSG) with interlaminated hybrid composite (100\% HSC-cSG): (a) Experimental and (b) Analytical model predicted stress-strain curves. 


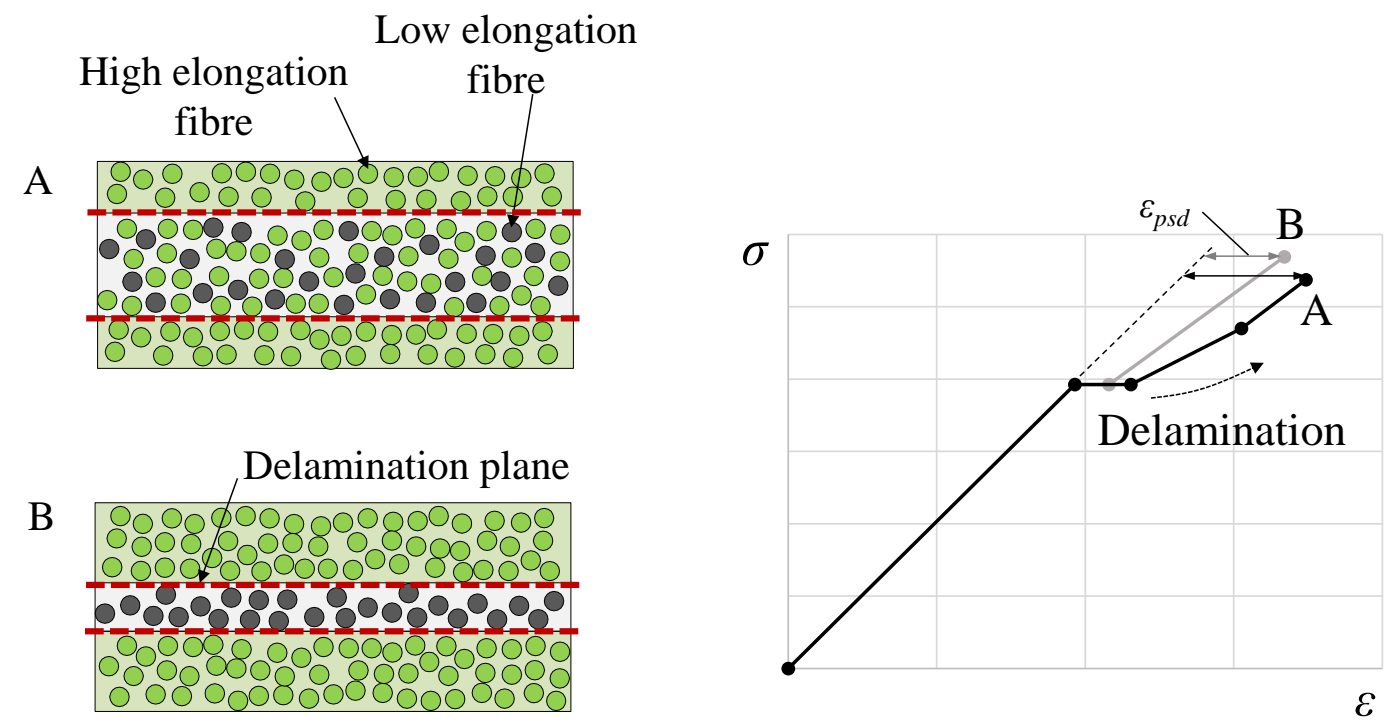

Low elongation fibre portion $\mathrm{A}=\mathrm{B}$

(a)

(b)

Figure 18. (a) Schematic diagram for comparison between hierarchical hybrids and interlaminated hybrids with two types of fibre, (b) their stress-strain curves. 